\title{
DISEÑO SÍSMICO DE MARCOS DE ACERO CONTRAVENTEADOS. PARTE 1: RECOMENDACIONES DE DISEÑO
}

\author{
Edgar Tapia Hernández ${ }^{(1)}$ y Arturo Tena Colunga ${ }^{(2)}$
}

\begin{abstract}
RESUMEN
A fin de contribuir al conocimiento adquirido sobre el diseño sísmico de edificios estructurados con marcos momento resistentes dúctiles de acero contraventeados, se presenta una metodología de diseño por capacidad adaptada al Reglamento de Construcciones para el Distrito Federal (RCDF04), que pretende establecer los requisitos mínimos que deben ser considerados en el diseño de este sistema estructural para que los mecanismos de colapso desarrollados concuerden con las premisas implícitas en el diseño (columna fuerte - viga débil - contraviento más débil), con una adecuada distribución de la respuesta inelástica en la altura y redistribuciones de cargas predecibles. La metodología, que incluye factores por ductilidad y sobrerresistencia más realistas para este sistema estructural, se ejemplifica en un modelo de 14 niveles diseñado en suelo blando.
\end{abstract}

Palabras clave: marcos dúctiles, contraventeo concéntrico, diseño por capacidad, ductilidad, factor de reducción por sobrerresistencia, desempeño estructural.

\begin{abstract}
In order to help improving the current knowledge for the seismic design of regular steel buildings structured with ductile moment - resisting concentrically braced frames (MRCBFs), a capacity design methodology adapted for the Mexican Federal District Code (MFDC-04) is presented. In the proposed methodology minimum requirements are established in order to insure collapse mechanisms consistent with the assumptions implicit in a code-based design (strong column, weak beam, and weaker brace), with a uniform distribution of yielding along the height and predictable load redistributions. Improved equations are proposed for a more realistic assessment of ductility and overstrength force modification factors for this structural system. In the paper, the proposed methodology is illustrated with the design of a 14-story building settled in soft soil conditions.
\end{abstract}

Keywords: ductile frames, concentric bracing, capacity design, ductility, overstrength factor, structural performance.

\section{INTRODUCCIÓN}

Conforme al Reglamento de Construcciones del Distrito Federal (RCDF-04), un edificio estructurado con marcos dúctiles contraventeados debe diseñarse considerando que en cada entrepiso los marcos son capaces de resistir, sin contar el sistema de contravientos, cuando menos el 50 por ciento de la

Artículo recibido el 4 de mayo de 2012 y aprobado para su publicación el 9 de enero de 2013.

(1) Profesor, Departamento de Materiales, Universidad Autónoma Metropolitana Azcapotzalco, Av. San Pablo 180, Col. Reynosa Tamaulipas, 02200 México, DF, e-mail: etapiah@correo.azc.uam.mx

(2) Profesor, Departamento de Materiales, Universidad Autónoma Metropolitana Azcapotzalco, Av. San Pablo 180, Col. Reynosa Tamaulipas, 02200 México, DF, e-mail: atc@correo.azc.uam.mx 
fuerza sísmica actuante. Además, en el procedimiento de diseño se supone que el sistema desarrollará un mecanismo de colapso columna fuerte - viga débil - contraviento más débil, si se siguen estrictamente las recomendaciones de diseño y detallado de los elementos de acero estipulados en las Normas Técnicas Complementarias para Diseño por Sismo (NTCS-04) y para Estructuras Metálicas (NTCM-04).

En investigaciones previas realizados por los autores (Tapia 2005, Tapia y Tena 2009) se estudió el comportamiento de edificios de 15 pisos estructurados con marcos de acero contraventeados ubicados en la zona IIIb (NTCS-04), usando los módulos de diseño de software comercial, suponiendo un comportamiento dúctil y siguiendo estrictamente los criterios del Reglamento (RCDF-04). Los resultados de éste y otros estudios (Tapia-Hernández y Tena-Colunga 2008) evidenciaron mecanismos de colapso de piso suave con articulaciones en ambos extremos de columnas en los pisos intermedios, a pesar de que los modelos fueron diseñados suponiendo un comportamiento dúctil, asociados con altas reservas de sobrerresistencia y formación de mecanismos de colapso por piso débil.

De hecho, en estudios recientes sobre el comportamiento de edificios estructurados con marcos contraventeados de acero (Marino y Nakashima 2005, Lacerte y Tremblay 2006, Izvernari 2007, GuiliniCharette 2009, Tapia 2011) se ha demostrado que aun siguiendo los lineamientos generales disponibles en reglamentos internacionales vigentes, los modelos no desarrollan forzosamente un comportamiento estructural adecuado ante sismos intensos, sino que su respuesta se puede ver dominada por la formación de mecanismos de colapso de pisos débiles, redistribuciones inesperadas de carga, concentración del daño, etc. El comportamiento inelástico de edificios estructurados con marcos contraventeados es dominado por la respuesta de las crujías contraventeadas, que es diferente al comportamiento de marcos momento resistentes sin contravientos.

Esta investigación presenta los resultados de un estudio en edificios estructurados con marcos momento resistentes de acero con contraventeo, a fin de mejorar el conocimiento adquirido y proponer una metodología de diseño por capacidad adaptada a los criterios Reglamento de Construcciones para el Distrito Federal (RCDF-04). Esta metodología pretende establecer los requisitos mínimos que deben ser considerados en el diseño de marcos dúctiles de acero con contraviento concéntrico para que los mecanismos de colapso desarrollados concuerden con las premisas iniciales de diseño de columna fuerte viga débil - contraviento más débil, con una adecuada distribución de la respuesta inelástica en la altura y redistribuciones de cargas predecibles.

En Tapia y Tena (2011) se analizaron edificios regulares estructurados con marcos dúctiles de acero con contraventeo concéntrico situados en suelo blando con alturas entre los 4 a los 16 niveles (14 m a 56 $\mathrm{m})$. Los modelos se diseñaron siguiendo estrictamente los criterios del RCDF-04 y variando la contribución al cortante lateral resistente de las columnas del marco en relación a la aportación del sistema de contravientos. Los resultados presentados en Tapia y Tena (2011), junto con planteamientos establecidos en reglamentos internacionales especializados, los resultados de pruebas experimentales y de otras investigaciones recientes, constituyen el fundamento de la metodología propuesta en este artículo.

Además, en este artículo se ejemplifica la aplicación de la metodología en un edificio regular de 14 pisos, que forma parte de un conjunto de modelos de 10, 14 y 18 niveles que se estudiaron para evaluar la metodología, cuyos resultados se discuten en el artículo complementario Tapia y Tena (2013).

\section{REQUISITOS GENERALES}

La metodología de diseño propuesta para marcos momento-resistentes dúctiles de acero con contraventeo concéntrico se muestra esquemáticamente en la figura 1. Como se aprecia, es necesario 
establecer el cortante lateral mínimo que debe ser resistido por las columnas del marco en relación a la aportación del sistema de contravientos conforme a resultados de investigaciones previas (Tapia 2011, Tapia y Tena 2011).

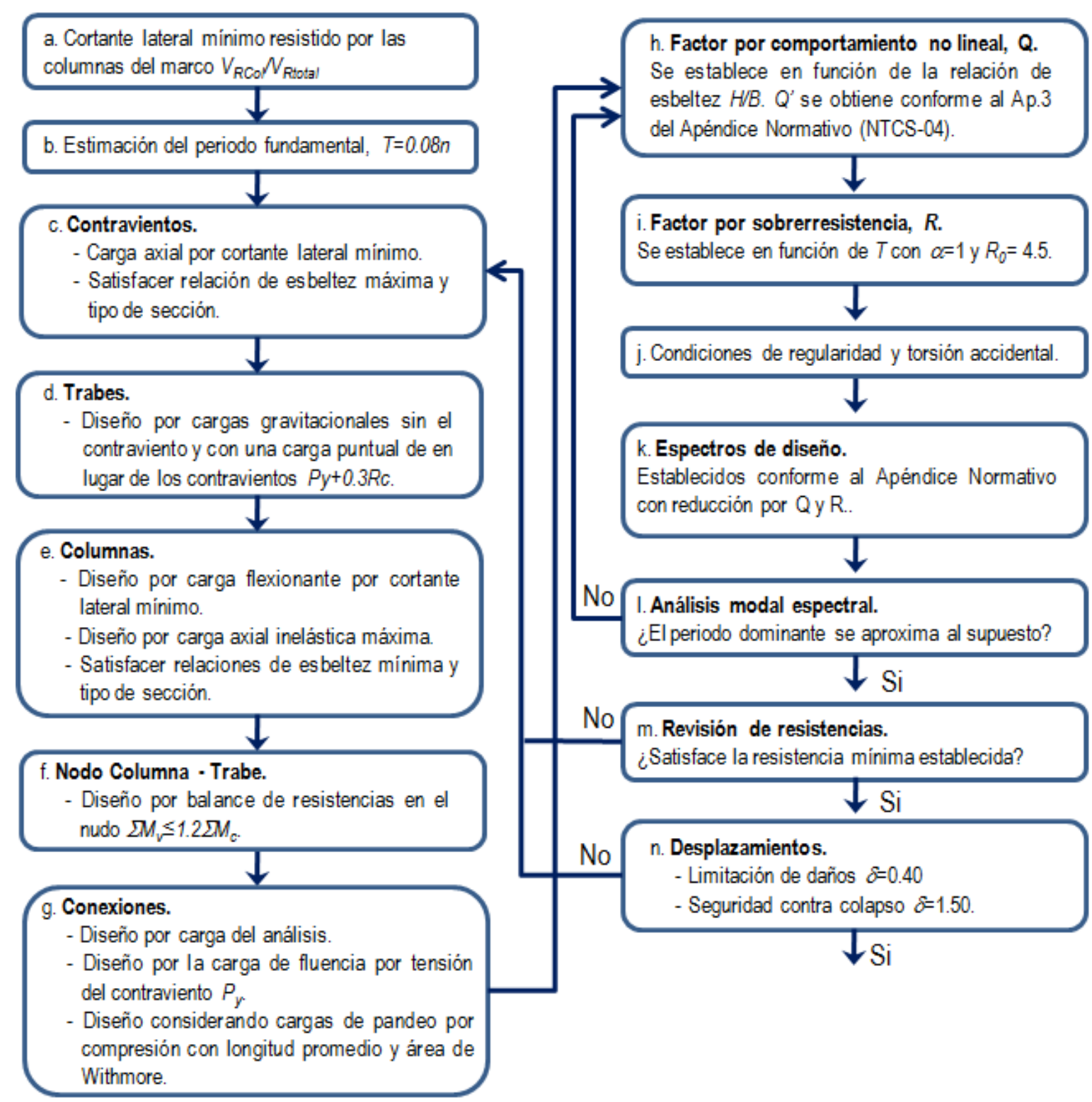

Figura 1. Diagrama propuesto de análisis y diseño de marcos de acero dúctiles con contravientos

\section{Cortante lateral mínimo resistido por las columnas}

Cada uno de los marcos rígidos que componen la estructura deberá diseñarse para resistir no menos que el porcentaje de fuerza lateral actuante determinado con la inecuación 1 (inciso $a$ de la figura 1), sin considerar la contribución del sistema de contravientos, que fue determinada, a partir de resultados presentados en Tapia y Tena (2011). 


$$
\frac{V_{R \text { Col }}}{V_{R \text { Total }}} \geq 0.50+0.05\left(\frac{H}{B}\right)^{2} \sqrt{\frac{F_{y \text { Col }}}{F_{y \text { Diag }}}}
$$

En la ecuación, $H$ es la altura del edificio, $B$ el ancho del edificio en la dirección estudiada, $F_{y c o l}$ el esfuerzo de fluencia de las columnas y $F_{y \text { Diag }}$ el esfuerzo de fluencia de los contravientos.

\section{Estimación del periodo fundamental}

En la metodología se propone estimar el periodo fundamental de la estructura de manera preliminar como $T=0.08 n$ conservadoramente, donde $n$ es el número de niveles del edificio (inciso $b$ de la figura 1 ). Esta fórmula se obtuvo a partir de un análisis de regresión de los periodos fundamentales (figura 2) de los diseños de los edificios de 4, 8, 12 y 16 niveles reportados en Tapia y Tena (2011), que satisfacen enteramente los requisitos del Reglamento de Construcciones para el Distrito Federal, y los resultados de diseño de dos edificios regulares de 12 y 15 pisos estructurados con marcos momento - resistentes de acero con contraventeo concéntrico que se estudiaron, analizaron y diseñaron en Tapia (2005).

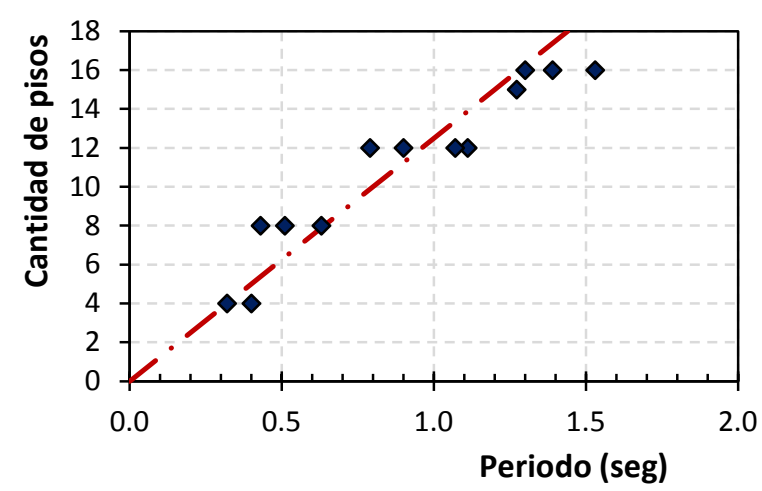

Figura 2. Evaluación de la propuesta para estimar el periodo fundamental

\section{Requisitos para contravientos}

Siguiendo la metodología propuesta, es necesario que los contravientos sean el primer grupo de elementos que sea diseñado (inciso $c$ de la figura 1). Los contravientos deben ser sección tipo 1 con una relación de esbeltez efectiva que cumpla $\frac{k L}{r}<5.88 \sqrt{E / F_{y}}$ como lo solicitan las Normas Técnicas (Ap. 6.2.3.2, NTCM-04), donde $k$ es el factor de longitud efectiva, $L$ la longitud libre, $r$ el radio de giro de la sección, $E$ es el módulo de elasticidad y $F_{y}$ el esfuerzo de fluencia. Cuando se requiera la carga axial postpandeo de los contravientos se podrá calcular como $0.3 R_{c}$ (Marino y Nakashima 2006).

Lacerte y Tremblay (2006), Izvernari et al. (2007) y Guilini-Charette (2009) presentaron estudios de marcos con contraventeo concéntrico de modelos entre los cuatro y dieciséis niveles, en los que se prestó especial atención en el diseño de las conexiones de cada uno de los entrepisos para cada condición particular. El objetivo de los estudios fue valorar el comportamiento de los edificios en análisis dinámicos no lineales considerando registros históricos y registros artificiales para suelos firmes, buscando mitigar la concentración de deformaciones inelásticas.

En los estudios referidos, una vez realizados los análisis, se diseñó cada una de las conexiones de los contravientos en cada entrepiso, lo que permitió estudiar la relación entre la longitud teórica $L_{\text {ejes }}$ de la 
intersección del contraviento en el nudo trabe - columna y la longitud efectiva del contraviento, $L_{\text {real }}$ (figura 3a). En la figura 3b se presentan los resultados de estas proporciones en modelos de ocho niveles, donde se graficó la relación entre la longitud real $L_{\text {real }}$ y la longitud a ejes $L_{\text {ejes }}$ del contraviento para cada conexión diseñada en cada uno de los entrepisos que conforman el modelo para ilustrar los datos disponibles.

El promedio de esta proporción resultó ser igual a $L_{\text {real }} / L_{\text {ejes }}=0.787$, de manera que los análisis que consideran la longitud de los contravientos de la distancia a ejes $\left(L_{\text {real }} / L_{\text {ejes }}=1.0\right)$ podrían liderar a resultados poco realistas, asociados a longitudes mayores y pandeos prematuros.

Por otra parte, con base en la experiencia adquirida en pruebas experimentales de contravientos tubulares en compresión, se sabe que mediante la aplicación de un factor de longitud efectiva $K=0.90$, se obtienen resultados analíticos similares a los obtenidos en las pruebas cuando los elementos son unidos con placas de conexión (Tremblay 2008). De esta forma, la proporción comentada decrecería hasta $L_{\text {real }} / L_{\text {ejes }}=0.709$. Es decir, que parece razonable considerar longitudes efectivas no arriostradas del contraviento cercanas al setenta por ciento de la longitud a ejes, con base en estos resultados.

a)

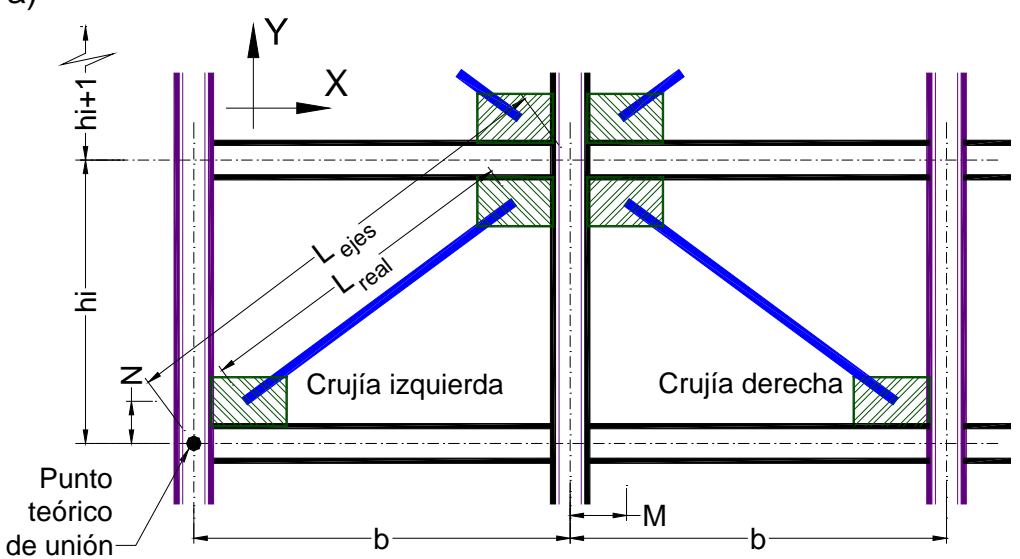

b)

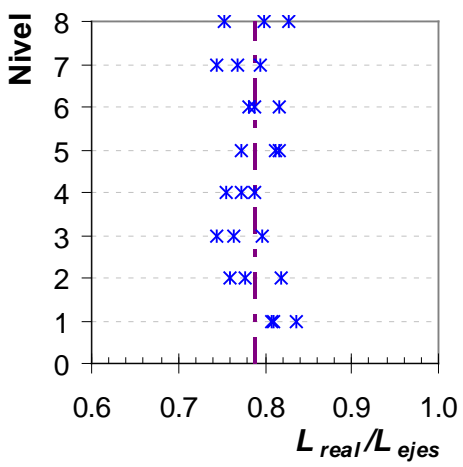

Figura 3. a) Longitud efectiva del contraviento, b) Proporciones obtenidas en Lacerte y Tremblay (2006) y Izvernari et al (2007) en modelos de ocho niveles

La importancia de esta premisa está asociada a la relación de esbeltez de los contravientos, $K L / r$, con fines de diseño y/o prediseño. Entre más robustos sean los contravientos (relaciones de esbeltez bajas) se esperan demandas de deformación plástica más altas antes del pandeo y posteriormente, cuando las rotaciones se desarrollan en los ciclos subsecuentes, se presenten con importantes deformaciones axiales (Tremblay 2008a). La falla de estos elementos ocurre cuando el contraviento es cargado en tensión, luego del pandeo desarrollado en previas incursiones de ciclos a compresión, por lo que para el diseñador debe resultar de especial interés determinar adecuadamente la esbeltez de los contravientos.

Este antecedente sobre la longitud real de los contravientos $L_{\text {real }}$ se consideró explícitamente en los análisis no lineales realizados, a partir de la reducción de la longitud a ejes del elemento y el diseño de las placas de conexión con la metodología presentada, a fin de obtener resultados que evalúen la aportación a compresión de los contravientos de la forma más realista y clara posible. 
Asimismo, se recomienda no hacer simultáneamente el cambio de sección transversal en columnas y contravientos en un mismo entrepiso. El procedimiento sugerido para el prediseño de los contravientos se ilustra más adelante en un edificio de 14 niveles.

\section{Requisitos para trabes}

Las secciones transversales de las trabes deberán ser tipo 1, sin cambios importantes o abruptos en la sección transversal de las vigas en las zonas de formación de articulaciones plásticas. Además, las vigas interceptadas por contravientos deben (inciso $d$ de la figura 1):

a. Ser continuas entre columnas.

b. Diseñarse para que resistan los efectos de todas las cargas tributarias, muertas y vivas, suponiendo que el contraviento no existe.

c. Diseñarse para que resistan los efectos de las cargas verticales, muertas y vivas, más una carga axial aplicada por las diagonales, calculada considerando una fuerza mínima igual a $P_{y}$ en la diagonal en tensión y una fuerza máxima de $0.3 R_{C}$ en la diagonal comprimida, donde $0.3 R_{C}$ representa la carga post-pandeo de los contravientos (Marino y Nakashima 2005). $P_{y}$ es la fuerza axial que ocasiona la plastificación $\left(P_{y}=A F_{y}\right)$ y $R_{c}$ la resistencia de diseño del contraviento en compresión axial y $F_{y}$ es el esfuerzo de fluencia y $A$ es el área transversal de la sección del contraviento.

\section{Requisitos para columnas}

Todas las columnas del marco deben ser continuas en al menos dos entrepisos, de sección transversal constante tipo 1 ó 2 y con una relación de esbeltez máxima de las columnas no debe exceder 60. Además, las columnas adyacentes a la crujía contraventeada deben (inciso $e$ de la figura 1):

a. Ser capaces de soportar un momento flexionante de al menos $0.2 Z F_{y}$ en combinación con la carga axial calculada en el análisis, donde $F_{y}$ es el esfuerzo de fluencia y $Z$ es el módulo de sección plástico.

b. Ser diseñada para soportar una carga axial $P_{k}$ determinada con la ecuación 2. Esta ecuación pretende considerar el aumento de carga axial que deberá resistir la columna por la plastificación de los contravientos en pandeo por compresión o fluencia por tensión. En Tapia et al. (2009) se presenta una comparación con otros métodos disponibles en la literatura para la determinación de incremento de carga axial por la respuesta inelástica de los contravientos y se incluyen mayores detalles sobre la determinación de esta ecuación:

$P_{k}=P_{g}+\operatorname{sen} \theta \sum_{n=i}^{i}\left[P_{f} f_{i}\right]$

en ella, $P_{g}$ es la carga axial asociada a las cargas gravitacionales que bajan por la columna $k, \theta$ es la inclinación de los contravientos con respecto al eje horizontal, $P_{f}$ la carga de fluencia de los contravientos, ya sea por pandeo en compresión o fluencia por tensión, y $f_{i}$ un factor de reducción que considera el porcentaje de contribución de la carga de fluencia de los contravientos a la columna del entrepiso $i$, cuya carga axial se está determinando.

El factor de reducción $f_{i}$ de la columna del entrepiso $k$ que evalúa el porcentaje de aportación de la carga de fluencia de los contravientos $P_{f}$ sigue el comportamiento propuesto en la ecuación 3. El factor $f_{i}$ es función del número total de niveles del edificio $S_{t}$, el entrepiso donde se encuentra 
la columna en la que se pretende determinar la carga axial $S_{n}$ y del entrepiso donde se está determinando el factor de reducción de la carga de fluencia de los contravientos $S_{i}$. En la ecuación mencionada, el subíndice $t$ indica el número total de pisos de la estructura, $i$ es el entrepiso donde se encuentra el contraviento para el que se está determinando el factor de reducción, y $n$ el entrepiso donde se encuentra la columna en la que se pretende determinar la carga axial.

$f_{i}=1-\frac{\left(S_{i}-S_{n}\right)^{2}}{0.9 S_{i}\left(S_{t}-S_{n}\right)}$

\section{Requisitos nudo trabe - columna}

Por tratarse de marcos dúctiles, se deberá también vigilar que, en cualquier nudo de interés, la suma de los momentos plásticos de las vigas así diseñadas $\left(\Sigma M_{v}\right)$ que llegan al nudo sea inferior a la suma de los momentos plásticos de las columnas $\left(\Sigma M_{c}\right)$ que llegan a ese nudo (inciso $f$ de la figura 1$)$, es decir:

$\sum M_{v} \leq \alpha \sum M_{c}$

La ecuación anterior debe cumplirse con $\alpha=1.2$, a diferencia de la propuesta vigente en la mayoría de los reglamentos internacionales (por ejemplo, Secc. 5.8.10, NTCM-04) que estipulan $\alpha=1.0$. Note que el planteamiento vigente en las NTC es poco conservador para estructuras que se diseñan para ser dúctiles, ya que permitir que la suma de los momentos plásticos de las vigas $\left(\Sigma M_{v}\right)$ sea igual a la suma de los momentos plásticos de las columnas $\left(\Sigma M_{c}\right)$ podría favorecer el desarrollo de mecanismos de colapso finales diferentes al supuesto desde la etapa de diseño: columna fuerte - viga débil.

En marcos de concreto reforzado con mecanismos columna fuerte - viga débil (-contraviento más débil), es necesario comprobar que el momento plástico de la viga es menor al momento plástico que resiste la columna para establecer condiciones de ductilidad local y global. Los reglamentos norteamericanos solicitan que el momento plástico resistente de la columna $M_{c}$ sea hasta $\alpha=1.5$ veces el momento plástico de la trabe $M_{v}$ (Godínez 2010), siendo el límite inferior $\alpha=1.3$ establecido en el reglamento europeo (Ap. 4.4.2.3.4, EC8-05).

De manera que esta propuesta $(\alpha=1.2)$ es congruente con lo estipulado para marcos momento resistentes dúctiles de concreto y pretende evitar la formación de mecanismos de colapso asociados al pandeo prematuro de las columnas. Además, se hace notar que considerar $\alpha=1.0$ es un criterio muy optimista y no favorece la formación de los mecanismos que se presuponen en la etapa elástica (columna fuerte - viga débil), sobre todo para diseñadores inexpertos que confíen demasiado en los módulos de diseño de programas comerciales. Entonces, en caso de que la ecuación 4 no se cumpla, se deberán rediseñar las secciones de las columnas y revisar que con las nuevas secciones se siga cumpliendo la inecuación 1.

\section{Requisitos en el diseño de la conexión}

La excentricidad en las conexiones de los contravientos debe ser siempre minimizada y se sugiere que la resistencia requerida sea la más pequeña de las siguientes, independientemente de la zona sísmica (inciso $g$ de la figura 1 ).

a. La resistencia nominal en tensión del elemento de contraventeo, calculada como $F_{y} A$.

b. La fuerza máxima determinada en el análisis. 
La resistencia en tensión debe ser la resistencia de diseño en tensión de los elementos de contraviento basada en los estados límite de fractura en la sección neta y de ruptura en el bloque por cortante y tensión, mientras que la resistencia en flexión en la dirección en la que se pandeará la diagonal debe ser mayor que la resistencia nominal esperada en flexión del contraventeo alrededor del eje de pandeo.

El diseño por tensión de la placa de conexión demanda la revisión de fractura en la sección neta y la ruptura en bloque por cortante (ecuación 5 y 6), a partir de la máxima carga a tensión que puede desarrollar el contraviento $F_{R} F_{y} A_{g}$, además de las posibles formas de pandeo. En las expresiones, $A_{t t}$ es el área total sujeta a tensión, $A_{t c}$ es el área total sujeta a cortante, $A_{n t}$ es el área neta sujeta a tensión, $A_{n c}$ es el área neta sujeta a cortante con $F_{R}=0.75$ (Ap. 5.4.3 NTCM-04).

$$
\begin{aligned}
& \text { Si } F_{u} A_{n t} \geq 0.60 F_{u} A_{n c} \\
& F_{R} R_{n}=F_{R}\left(0.6 F_{y} A_{t c}+F_{u} A_{n t}\right) \leq F_{R}\left(0.6 F_{u} A_{n c}+F_{u} A_{n t}\right) \\
& \text { Si } F_{u} A_{n t}<0.60 F_{u} A_{n c} \\
& F_{R} R_{n}=F_{R}\left(0.6 F_{u} A_{n c}+F_{y} A_{t t}\right) \leq F_{R}\left(0.6 F_{u} A_{n c}+F_{u} A_{n t}\right)
\end{aligned}
$$

Los contravientos y sus conexiones deben ser diseñados para que se forme alguna de las tres posibles articulaciones: en el centro y sus extremos. De esta forma, las conexiones deben ser diseñadas para que el pandeo ocurra en la placa o en el contraviento. Si la rotación ocurre en el contraviento, la conexión debe ser lo suficientemente fuerte para soportar las repeticiones de fuerza de manera dúctil (Cochran y Honeck 2004, Astaneh-Asl et al. 2006).

En contravientos esbeltos se espera un pandeo temprano, de modo que la placa de conexión se debe detallar para reacomodar la dirección del pandeo, por lo que la placa de conexión es más dependiente de la fuerza de tensión del contraviento $\left(A F_{y}\right)$. En cambio, cuando el contraviento es robusto, la capacidad adicional a compresión del contraviento debe considerarse para determinar el diseño de la placa y prevenir su pandeo. En ese sentido, la placa de conexión puede seguir dos criterios de diseño en función del pandeo: en el plano del marco o fuera del plano, por lo que en el diseño de las placas de conexión debe considerarse sus posibles formas de pandeo, mediante una distribución de esfuerzos uniforme con una longitud promedio y el área de la sección de Whitmore, como se explicará más adelante.

Entonces, es de importancia en el diseño de la placa de conexión considerar el plano de pandeo en el plano del marco o fuera del mismo (Haddad y Tremblay 2006). En el caso específico de conexiones diseñadas cuando el pandeo es fuera del plano, se debe considerar el eje de pandeo de la placa de conexión perpendicular al eje del contraviento. Se recomienda que el contraviento sea cortado a una distancia de dos veces el espesor de la placa de conexión para la formación del eje de pandeo (Cochran y Honeck 2004).

El diseño por compresión de la placa se realiza a partir de la sección Whitmore propuesta en 1952, definida como el ancho efectivo de la placa de conexión perpendicular al eje del elemento e interceptado por dos líneas a 30 grados (figura 4). A partir de este ancho, que considera una distribución de los esfuerzos uniforme, se obtienen tres posibles longitudes de pandeo $L_{g 1}, L_{g 2}$ y $L_{g 3}$, que dependen de las características geométricas de la inclinación del contraviento, y que se definen considerando un ángulo de 30 grados y la longitud de la soldadura $L_{w}$. Siguiendo este planteamiento, a partir de esas longitudes se define la longitud de pandeo promedio $\left(L_{g 1}+L_{g 2}+L_{g 3}\right) / 3$ asociada al área de la sección de Whitmore 
(perpendicular al eje del contraviento). Además, la figura 4a incluye las holguras recomendadas en el eje paralelo y perpendicular al contraviento para la formación del área de Whitmore.

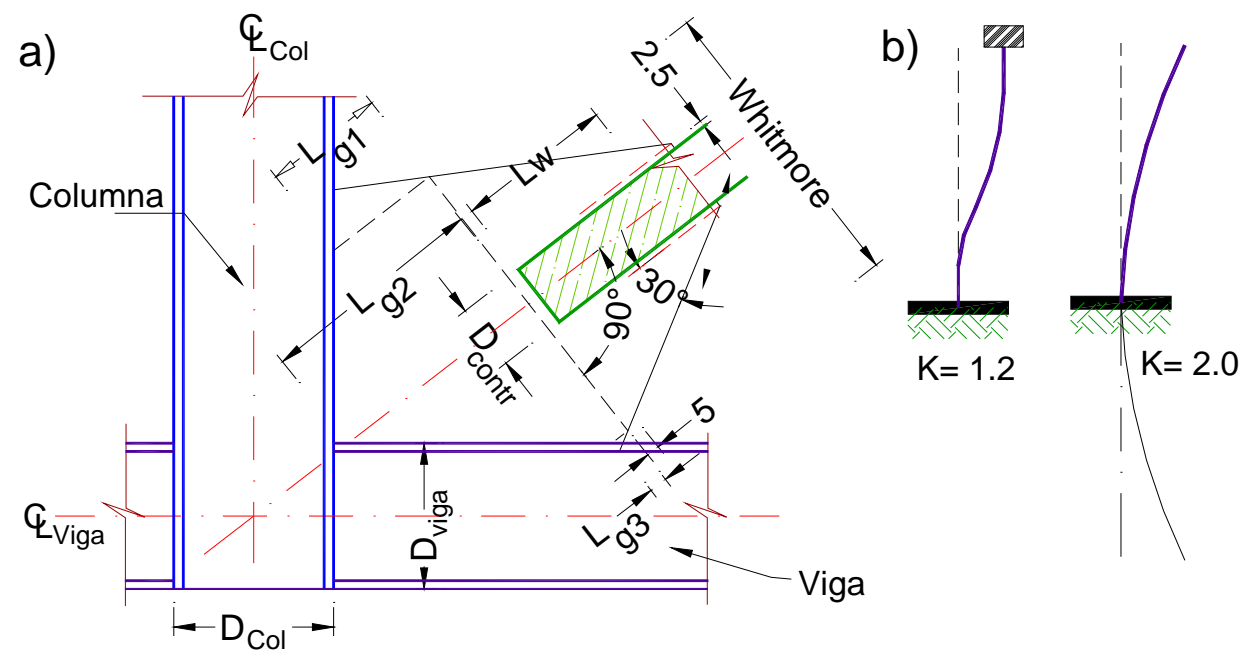

Figura 4. Consideraciones geométricas en la conexión contraviento - viga - columna

Respecto a la relación de esbeltez para la revisión por compresión de la placa de conexión, en este estudio se aceptó la práctica común (figura $4 \mathrm{~b}$ ), que considera un factor de longitud efectivo igual a $K=1.2$ (Cochran y Honeck 2004, Izvernari 2007), aunque existen estudios que proponen $K=2.0$, por considerar que la placa se encuentra fija en un extremo y completamente libre en el otro (Tremblay 2008).

Entonces, la placa de conexión se diseña considerando una longitud de la articulación al menos igual a dos veces el espesor de la placa (2t), aunque una práctica común es considerar dos pulgadas (5 $\mathrm{cm})$, como se ilustra en la figura $4 \mathrm{a}$.

Finalmente, es valioso comentar que en algunas publicaciones como en Cochran y Honeck (2004) y Lundeen (2003) se recomienda considerar placas de conexión rígidas con aceros de alta resistencia (A-572 Gr.50) por dos motivos: a) para asegurar que la conexión soportará la sobrerresistencia del contraviento en tensión y b) reducir el espesor de la placa en comparación con lo que se obtendría con placas de acero A36.

En contravientos esbeltos, cuando se espera un pandeo temprano, la placa se debe detallar para reacomodar las fuerzas en la dirección del pandeo, de modo que el diseño de la placa de conexión es más dependiente de la fuerza de tensión del contraviento $A_{g} F_{y} F_{R}$, mientras que en contravientos robustos, la capacidad adicional a compresión del contraviento debe considerarse para determinar el diseño de la placa de conexión y prevenir su pandeo prematuro (Astaneh-Asl et al. 2006).

Cuando por procedimientos constructivos o estéticos la conexión esté embebida en el concreto en una profundidad menor a $45 \mathrm{~cm}$, no se recomienda hacer una reducción de la longitud de pandeo, ya que se ha demostrado que no se desarrolla la suficiente longitud de empotramiento (Cochran y Honeck 2004).

Durante un sismo, el comportamiento de la conexión se complica por fuerzas generadas por deformaciones por distorsión. Cuando el marco se distorsiona horizontalmente debido a la carga lateral, la configuración varía a un paralelogramo que origina tensión en la placa que sufre pandeo por compresión 
debido a que el ángulo inicial ortogonal entre la viga y la columna se reduce. Este efecto, que no se considera en los reglamentos vigentes (Cochran y Honeck 2004), no debe despreciarse en el diseño de placas de conexión delgadas.

\section{DISPOSICIONES PARA EL DISEÑO SÍSMICO}

Para realizar los análisis sísmicos se deberá considerar explícitamente los efectos del periodo dominante más largo del terreno, $T_{s}$. Las ordenadas espectrales de aceleración se obtendrán con el planteamiento del Apéndice A (NTCS-04) y podrán ser reducidas por los factores de ductilidad Q' y de sobrerresistencia $R$ específicos para este sistema estructural conforme a las siguientes secciones.

\section{Factor de reducción por ductilidad}

Los marcos dúctiles con contraventeo concéntrico tienen la capacidad de formar articulaciones plásticas donde sean necesarias y mantener su resistencia en dichas articulaciones, disipando energía mediante la fluencia de las diagonales o de sus conexiones. Para aplicar los factores de reducción por ductilidad será necesario que, además de que sus columnas, trabes, contravientos y conexiones satisfagan los requisitos comentados anteriormente, cumplan los siguientes requisitos:

a. La resistencia en todos los entrepisos es suministrada exclusivamente por marcos contraventeados en los que en cada entrepiso los marcos son capaces de resistir, sin contar el sistema de contravientos, cuando menos el porcentaje de la fuerza sísmica actuante determinado mediante la ecuación 1.

b. Los marcos rígidos de acero satisfacen los requisitos para marcos con ductilidad alta que fijan las Normas correspondientes.

Conforme a la apartado 6.23 sobre "Marcos con contraventeo concéntrico dúctil” de las NTCS-04, los marcos con contraventeo concéntrico dúctil se definen como aquéllos que tienen la capacidad de disipar energía mediante la fluencia de las diagonales o de sus conexiones; sin embargo, después de la fluencia de las diagonales, es necesario que el sistema de trabes y columnas sigan evidenciando un comportamiento dúctil aunque algunos de los contravientos ya hayan fluido para que el sistema estructural en su conjunto sea dúctil. Esto es, en las NTCS a) no se aclara que el marco (conexiones, trabes y columnas) debe diseñarse también como un sistema dúctil y, b) el apartado 6.23 presupone que los marcos con contraventeados tendrán una adecuada distribución de la respuesta inelástica en la altura del edificio, pero no explican cómo lograrlo; no establece los criterios para evitar la formación de un mecanismo de piso débil.

Entonces, el inciso $a$ pretende establecer los criterios para conseguir una adecuada distribución en la altura de la respuesta inelástica asociándola a un mecanismo de colapso columna fuerte - viga débil contraviento más débil mediante la aplicación de la ecuación 1 y, puesto que se espera la falla del contraviento como primera línea de defensa, mediante el inciso $b$ se pretende conseguir que la estructura sea capaz de comportarse en todos los casos como un sistema dúctil.

Asimismo, se deberán vigilar las especificaciones de las Normas Técnicas referentes a las Condiciones de regularidad (Ap. 6, NTCS-04) y los métodos de análisis estáticos y dinámicos (Ap. 8 y 9, NTCS-04).

En caso que se cumplan los requisitos anteriores (conforme al inciso $h$ de la figura 1 ), el factor de reducción por ductilidad se determina mediante las siguientes desigualdades (ecuación 7), donde $H$ es la 
altura y $B$ el ancho el ancho del edificio en la dirección global en la que se está realizando el análisis. La ecuación considera que la ductilidad que pueden desarrollar los modelos decrece con el aumento de la relación de esbeltez del edificio $H / B$, con base en resultados de estudios previos que pueden ser consultados en Tapia y Tena (2011).

$$
\text { Si }\left\{\begin{array}{rlrl}
\frac{H}{B} & \leq 1 & Q & =3 \\
1<\frac{H}{B} & \leq 1.6 & Q & =3-\frac{5}{3}\left(\frac{H}{B}-1\right) \\
\frac{H}{B}>1.6 & Q & =2
\end{array}\right.
$$

El comportamiento de la ecuación anterior se ilustra en la figura 5, en relación a la propuesta vigente del RCDF-04 y del Reglamento Canadiense (CNBC-05), que es el único de los reglamentos comparados en Tapia y Tena (2011) que reconoce que la ductilidad en los edificios no puede permanecer constante entre modelos de baja altura y modelos altos, sino que debe haber un decremento en las características del comportamiento no lineal esperado por los edificios.

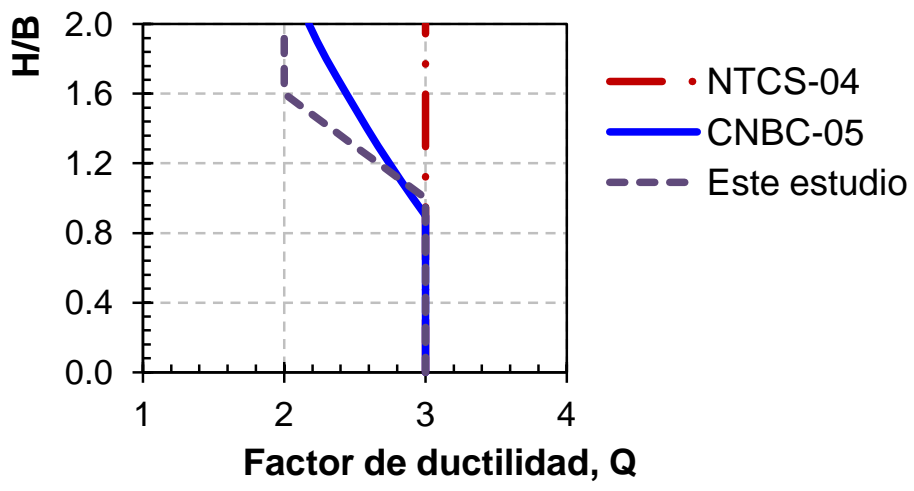

Figura 5. Comportamiento del factor de ductilidad propuesto en relación a otros reglamentos

Además, todas las estructuras deberán atender los requisitos para la clasificación de la edificación en regular, irregular o fuertemente irregular atendiendo a las previsiones que éstas impliquen.

\section{Factor de reducción por sobrerresistencia}

El factor por sobrerresistencia (inciso $i$ de la figura 1) se define mediante las siguientes desigualdades (ecuación 8), a partir de los resultados publicados en Tapia y Tena (2011), donde $R_{0}=4.5 \mathrm{y}$ $\alpha=1$ para marcos de acero contraventeados, $T_{a}$ es el periodo característico determinado en función del periodo dominante del terreno $T_{s}$, el primer periodo de la estructura $T$.

$$
\begin{array}{cc}
\text { Si } T \leq T_{a} & R=R_{0}+\alpha\left(1-\sqrt{\frac{T}{T_{a}}}\right) \\
\text { Si } T>T_{a} & R=R_{0}
\end{array}
$$


En la figura 6b se presenta el comportamiento de la ecuación 8 para distintos periodos dominantes del suelo, mientras que en la figura 6a, se ilustra el comportamiento de la propuesta de determinación del factor por sobrerresistencia conforme al RCDF vigente.

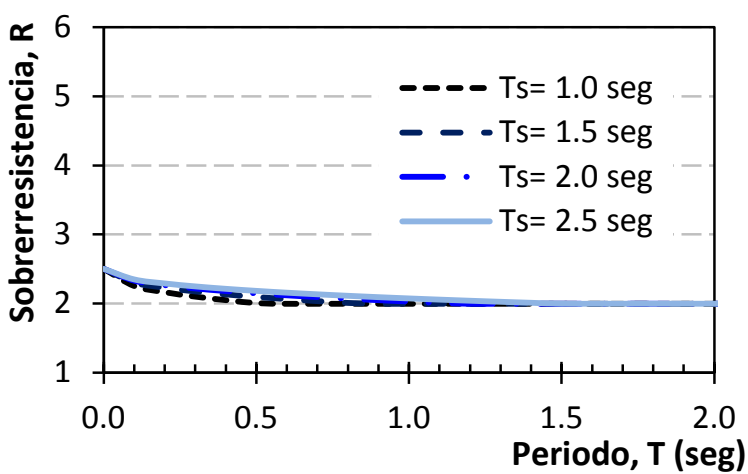

a) Determinación conforme a las NTCS-04

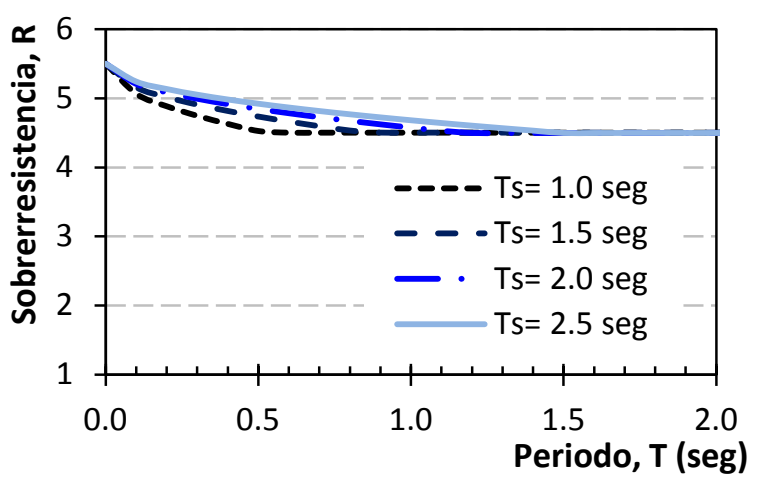

b) Determinación conforme a este estudio

Figura 6. Comportamiento del factor por sobrerresistencia en función del periodo dominante del suelo

Es valioso hacer notar que las NTCS-04 proponen una única metodología para determinar la reducción por sobrerresistencia en el Apéndice A (A.3 NTC-04), que es aplicable a todos los sistemas estructurales, sin importar el material, configuración, etc. Específicamente, la propuesta de este estudio para marcos dúctiles de acero contraventeado toma en cuenta los resultados de investigaciones recientes que reportan sobrerresistencias significativamente más altas que las propuestas en las NTC (Tapia 2011y Tapia y Tena 2011). Esta metodología (figura 6b) pretende evaluar la sobrerresistencia que pueden desarrollar marcos de acero contraventeados como se discute en Tapia y Tena (2011), con una magnitud máxima igual a $R=5.5$ en modelos de baja altura.

En cambio, el planteamiento de las NTCS-04 no distingue la dependencia de la sobrerresistencia que pueden desarrollar las estructuras en función del sistema estructural y/o del material utilizado, de manera que propone una única ecuación general con magnitudes máximas igual a $R=2.5$. En Tapia y Tena (2011) se presenta una discusión más profunda de la obtención y justificación de estas magnitudes con base en estudios recientes y propuestas de reglamentos internacionales.

La evaluación de la metodología propuesta se presenta en el artículo complementario Tapia y Tena (2013), donde se exponen los resultados de análisis inelásticos realizados a edificios de 10, 14 y 18 niveles ubicados en la zona IIIa, que fueron diseñados con la metodología de diseño por capacidad adaptada al RCDF-04, expuesta en este artículo. Con la finalidad de ilustrar su aplicación, en la siguiente sección se emplea el procedimiento de diseño propuesto en un modelo de 14 niveles.

\section{DESARROLLO DE LOS ANÁLISIS}

En todos los casos será necesario verificar las condiciones de regularidad y torsión accidental de los edificios (inciso $j$ de la figura 1) para afectar, en caso que sea necesario, el factor de reducción por comportamiento sísmico con fines de diseño $Q$ ’ por los factores correctivos correspondientes, conforme lo estipulan las Normas Técnicas (Ap. 6.4 NTCS-04). 
Los espectros de diseño se obtienen aplicando los criterios del Apéndice A (NTCS), donde los espectros inelásticos de diseño deberán considerar los factores de reducción por ductilidad con fines de diseño $Q$ ’ y sobrerresistencia $R$ que se proponen en esta investigación (inciso $k$ de la figura 1 ).

Entonces, una vez que se efectúe el análisis modal espectral, es necesario verificar que el periodo fundamental de la estructura se aproxime al periodo supuesto (inciso $l$ de la figura 1), de manera que en caso que sea necesario, se deberán recalcular los factores de reducción por sobrerresistencia y ductilidad y, por consiguiente, el espectro de diseño.

Finalmente, será necesario verificar que las secciones estructurales propuestas satisfacen el cortante lateral mínimo que debe ser resistido por las columnas del marco $V_{R C o l} / V_{R T o t a l}$ (inciso $m$ de la figura 1). En caso que no se cumplan, será necesario rediseñar los elementos que conforman la estructura, es decir, regresar hasta el inciso $c$ de la figura 1. Además, se solicita que la estructura cumpla las restricciones de deformación que se estipulan en el Apéndice A (inciso $n$ de la figura 1).

\section{DESCRIPCIÓN DEL MODELO}

A fin de ejemplificar el procedimiento, se diseñó un modelo de catorce niveles con la configuración de entrepiso tipo mostrada en la figura 7, conformado por 36 columnas en cada entrepiso separadas a 7 metros de manera que la planta es de $35 \mathrm{~m}$ x $35 \mathrm{~m}$ con alturas de entrepiso de $3.50 \mathrm{~m}$. Las columnas son de sección cajón con acero A-572 Gr.50, con trabes de sección I de acero A-36 y seis pares de contravientos en cada dirección global con configuración chevrón de sección cajón con acero A-36.

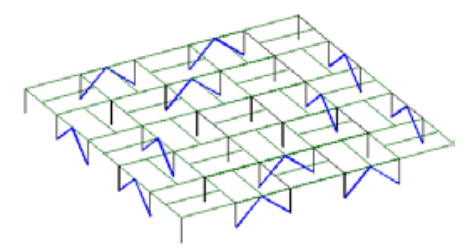

Figura 7. Isométrico del entrepiso tipo del modelo estudiado

En la tabla 1 se estipulan las combinaciones de carga consideradas en los análisis, mientras que la magnitud de las cargas se determinó conforme a lo que se explica en los siguientes apartados.

\section{Cargas gravitacionales}

En la tabla 2 se reportan las cargas gravitacionales consideradas. La determinación de la carga muerta incluye el peso del sistema de piso, cancelería, loseta, instalaciones, etc., siendo que la carga asociada al peso de los elementos estructurales se determinó internamente con el programa de análisis empleado.

En lo referente a la carga viva, se aceptaron los valores considerados para edificios de oficinas en las Normas Técnicas (NTCCr-04), donde $W_{a}$ es la carga viva instantánea y $W_{m}$ es la carga viva máxima, respectivamente. 


\section{PREDISEÑO DE ELEMENTOS ESTRUCTURALES}

El cortante lateral resistente mínimo que deben soportar las columnas asciende al 61 por ciento en relación a la aportación del sistema de contravientos conforme a la inecuación 1 , donde para el modelo ejemplificado $F_{y \text { Diag }}=2,530 \mathrm{~kg} / \mathrm{cm}^{2}, F_{y \text { Col }}=3,515 \mathrm{~kg} / \mathrm{cm}^{2}$ y $H / B=1.4$. El detalle de las aportaciones necesarias al cortante lateral de cada entrepiso por cada sistema estructural, a partir de las cargas determinadas con el análisis estático se presenta en la tabla 3 (inciso a de la figura 1). Conforme a la propuesta el periodo de la estructura aproximado es $T=0.08 n=1.12 \mathrm{seg}$ (inciso $b$ de la figura 1 )

Tabla 1. Combinaciones de carga considerada en los análisis

\begin{tabular}{cccccc}
\hline Comb. & $\begin{array}{c}\text { Carga } \\
\text { muerta }\end{array}$ & $\begin{array}{c}\text { Carga viva } \\
\text { máxima }\end{array}$ & $\begin{array}{c}\text { Carga viva } \\
\text { accidental }\end{array}$ & $\begin{array}{c}\text { Sismo } \\
\text { en X }\end{array}$ & $\begin{array}{c}\text { Sismo } \\
\text { en Y }\end{array}$ \\
\hline 1 & 1.40 & 1.40 & 0.00 & 0.00 & 0.00 \\
2 & 1.10 & 0.00 & 1.10 & 1.10 & 0.33 \\
3 & 1.10 & 0.00 & 1.10 & 1.10 & -0.33 \\
4 & 1.10 & 0.00 & 1.10 & -1.10 & 0.33 \\
5 & 1.10 & 0.00 & 1.10 & -1.10 & -0.33 \\
6 & 1.10 & 0.00 & 1.10 & 0.33 & 1.10 \\
7 & 1.10 & 0.00 & 1.10 & -0.33 & 1.10 \\
8 & 1.10 & 0.00 & 1.10 & 0.33 & -1.10 \\
9 & 1.10 & 0.00 & 1.10 & -0.33 & -1.10 \\
\hline
\end{tabular}

Tabla 2. Carga considerada en los modelos $\left(\mathrm{kg} / \mathrm{m}^{2}\right)$

\begin{tabular}{lccc}
\hline \multirow{2}{*}{ Nivel } & Carga muerta & \multicolumn{2}{c}{ Carga viva } \\
\cline { 2 - 4 } & $W_{C M}$ & $W_{a}$ & $W_{m}$ \\
\hline Entrepiso & 390 & 180 & 250 \\
Azotea (con pendiente menor al 5\%) & 325 & 70 & 100 \\
\hline
\end{tabular}

Tabla 3. Cortante lateral resistente que debe soportar cada sistema sismorresistente

\begin{tabular}{|c|c|c|c|}
\hline Nivel & $\begin{array}{c}\text { Cortante } \\
\text { estático } \\
\text { (ton) }\end{array}$ & $\begin{array}{l}\text { Cortante por } \\
\text { columnas } \\
\text { (61.6\%) } \\
\text { (ton) }\end{array}$ & $\begin{array}{l}\text { Cortante por } \\
\text { contravientos } \\
\text { (38.4\%) } \\
\text { (ton) }\end{array}$ \\
\hline 14 & 173.3 & 106.7 & 66.5 \\
\hline 13 & 405.5 & 249.8 & 155.7 \\
\hline 12 & 619.8 & 381.8 & 238.0 \\
\hline 11 & 816.3 & 502.8 & 313.5 \\
\hline 10 & 994.9 & 612.9 & 382.0 \\
\hline 9 & $1,155.7$ & 711.9 & 443.8 \\
\hline 8 & $1,298.5$ & 799.9 & 498.6 \\
\hline 7 & $1,423.6$ & 876.9 & 546.7 \\
\hline 6 & $1,530.7$ & 942.9 & 587.8 \\
\hline 5 & $1,620.0$ & 997.9 & 622.1 \\
\hline 4 & $1,691.5$ & $1,042.0$ & 649.5 \\
\hline 3 & $1,745.1$ & $1,075.0$ & 670.1 \\
\hline 2 & $1,780.8$ & $1,097.0$ & 683.8 \\
\hline 1 & $1,798.7$ & $1,108.0$ & 690.7 \\
\hline
\end{tabular}




\section{Prediseño del sistema de contravientos}

Conforme a la metodología propuesta (inciso $c$ de la figura 1), una vez que se definió el cortante lateral resistente máximo que debe aportar el sistema de contravientos, su sección transversal de prediseño se determina a través de la capacidad resistente (ecuación 9), que considera la fluencia por tensión y el pandeo por compresión por cada par de contravientos $m$.

$V_{k \text { contr }}=m F_{R} A_{t} F_{y} \cos \theta\left(1+\frac{1}{\left(1+\lambda^{2 n}\right)^{1 / n}}\right)$

En la ecuación anterior, $m$ es el número de pares de contravientos en la dirección estudiada en el entrepiso $k, F_{R}$ el factor de resistencia igual a $0.90, A_{t}$ el área transversal del contraviento, $F_{y}$ el esfuerzo de fluencia de los contravientos, $\theta$ el ángulo de inclinación de los contravientos, $n$ un parámetro igual a 1.4 y $\lambda$ un parámetro de esbeltez que se calcula conforme a las NTCM-04 con un factor de longitud efectiva del contraviento igual a $k L_{\text {ejes }}=0.709 L_{\text {ejes }}$, antes discutido.

La sección transversal del contraviento por entrepiso que satisface el máximo cortante lateral resistente (tabla 3 ) se presenta en la tabla 4, donde $d$ es el peralte de la sección cajón, $t$ el espesor de las placas, la longitud del contraviento es $495 \mathrm{~cm}$, con $m=6$ contravientos por cada dirección global y $\theta=45^{\circ}$. En la determinación del tipo de sección y la capacidad resistente a compresión y tensión se consideró lo dispuesto en las Normas de Estructuras Metálicas del RCDF-04 con $n=1.4$ y $F_{y}=2,530 \mathrm{~kg} / \mathrm{cm}^{2}$.

Tabla 4. Prediseño de contravientos del modelo de 14 niveles

\begin{tabular}{cccccccccc}
\hline Nivel & $\begin{array}{c}\text { Sección propuesta } \\
d\end{array}$ & $\begin{array}{c}t \\
(\mathrm{~cm})\end{array}$ & $\begin{array}{c}\text { Tipo de } \\
\text { sección }\end{array}$ & $\begin{array}{c}A_{t} \\
\left(\mathrm{~cm}^{2}\right)\end{array}$ & $K L / r$ & $\lambda$ & $\begin{array}{c}\text { Compresión } \\
P_{R} \\
(\mathrm{~kg})\end{array}$ & $\begin{array}{c}\text { Tensión } \\
T_{R} \\
(\mathrm{~kg})\end{array}$ & $\begin{array}{c}\text { Aportación total } \\
m\left(P_{R}+T_{R}\right) \operatorname{sen} \theta \\
(\mathrm{Ton})\end{array}$ \\
\hline 14 & 18 & 0.48 & 1 o 2 & 33.64 & 69.2 & 0.78 & 57,718 & 76,595 & 569.8 \\
13 & 18 & 0.48 & 1 o 2 & 33.64 & 69.2 & 0.78 & 57,718 & 76,595 & 569.8 \\
12 & 18 & 0.64 & 1 o 2 & 44.44 & 69.8 & 0.78 & 75,805 & 101,194 & 750.9 \\
11 & 18 & 0.64 & 1 o 2 & 44.44 & 69.8 & 0.78 & 75,805 & 101,194 & 750.9 \\
10 & 18 & 0.64 & 1 o 2 & 44.44 & 69.8 & 0.78 & 75,805 & 101,194 & 750.9 \\
9 & 18 & 0.64 & 1 o 2 & 44.44 & 69.8 & 0.78 & 75,805 & 101,194 & 750.9 \\
8 & 18 & 0.79 & 1 o 2 & 54.38 & 70.4 & 0.79 & 92,244 & 123,831 & 916.7 \\
7 & 18 & 0.79 & 1 o 2 & 54.38 & 70.4 & 0.79 & 92,244 & 123,831 & 916.7 \\
6 & 18 & 0.79 & 1 o 2 & 54.38 & 70.4 & 0.79 & 92,244 & 123,831 & 916.7 \\
5 & 18 & 0.79 & 1 o 2 & 54.38 & 70.4 & 0.79 & 92,244 & 123,831 & 916.7 \\
4 & 18 & 0.95 & 1 o 2 & 64.79 & 71.0 & 0.80 & 109,228 & 147,527 & $1,089.3$ \\
3 & 18 & 0.95 & 1 o 2 & 64.79 & 71.0 & 0.80 & 109,228 & 147,527 & $1,089.3$ \\
2 & 18 & 0.95 & 1 o 2 & 64.79 & 71.0 & 0.80 & 109,228 & 147,527 & $1,089.3$ \\
1 & 18 & 0.95 & 1 o 2 & 64.79 & 71.0 & 0.80 & 109,228 & 147,527 & $1,089.3$ \\
\hline
\end{tabular}

\section{Prediseño de trabes por cargas gravitacionales}

En el diseño de las secciones transversales de las trabes principales se tomaron en cuenta los criterios presentados en este artículo (inciso $d$ de la figura 1), de manera que fue necesario realizar un análisis adicional en el programa de análisis empleado con cargas puntuales que simulan la plastificación de los contravientos por tensión $P_{y}$ y la carga post-pandeo de los contravientos en compresión $0.3 P_{R}$, cuyas magnitudes se presentan en la tabla 5. 
Tabla 5. Determinación de carga puntual sobre las trabes de las crujías contraventeadas

\begin{tabular}{ccccc}
\hline Nivel & $\begin{array}{c}A_{\mathrm{t}} \\
\left(\mathrm{cm}^{2}\right)\end{array}$ & $\begin{array}{c}P_{\mathrm{y}} \\
(\mathrm{kg})\end{array}$ & $\begin{array}{c}0.3 P_{\mathrm{R}} \\
(\mathrm{kg})\end{array}$ & $\begin{array}{c}\left(P_{y}+0.3 P_{R}\right) \operatorname{sen} \theta \\
(\mathrm{kg})\end{array}$ \\
\hline 14 & 33.64 & 76,595 & 17,315 & 12,244 \\
13 & 33.64 & 76,595 & 17,315 & 12,244 \\
12 & 44.44 & 101,194 & 22,742 & 16,081 \\
11 & 44.44 & 101,194 & 22,742 & 16,081 \\
10 & 44.44 & 101,194 & 22,742 & 16,081 \\
9 & 44.44 & 101,194 & 22,742 & 16,081 \\
8 & 54.38 & 123,831 & 27,673 & 19,568 \\
7 & 54.38 & 123,831 & 27,673 & 19,568 \\
6 & 54.38 & 123,831 & 27,673 & 19,568 \\
5 & 54.38 & 123,831 & 27,673 & 19,568 \\
4 & 64.79 & 147,527 & 32,768 & 23,171 \\
3 & 64.79 & 147,527 & 32,768 & 23,171 \\
2 & 64.79 & 147,527 & 32,768 & 23,171 \\
1 & 64.79 & 147,527 & 32,768 & 23,171 \\
\hline
\end{tabular}

\section{Prediseño de las columnas}

Por otra parte, el prediseño de las 36 columnas por entrepiso se realizó asignando una sección transversal a las 20 columnas perimetrales y otra a las 16 columnas internas asociadas a mayor carga axial. Así, la sección transversal del prediseño se rige por las columnas adyacentes a las crujías contraventeadas y debe cumplir las siguientes dos condiciones (inciso $e$ de la figura 1):

a. Diseño por flexión, que considera la solicitación asociada al cortante lateral resistente mínimo determinado con la inecuación 1 (tabla 3).

b. Diseño por carga axial, que adiciona a las cargas axiales una componente de carga axial asociada a la respuesta inelástica de los contravientos por fluencia en tensión o pandeo en compresión.

c. Revisión por pandeo local y relaciones de esbeltez mínima.

\section{Prediseño por flexión de las columnas internas}

La contribución mínima que deben aportar las columnas al cortante lateral resistente se determina con la ecuación 10, igualando el momento plástico resistente $M_{R}=F_{R} Z F_{y}$ al momento producido por cortante lateral resistente necesario de la ecuación 1. De manera que el módulo de sección plástico necesario se determina como sigue:

$Z=\frac{V_{R \mathrm{Col}} h_{k}}{m F_{y} F_{R}}$

En la ecuación, $m$ es la cantidad de columnas del entrepiso $k, h$ la altura del entrepiso, $F_{y}$ el esfuerzo de fluencia del acero de las columna, $F_{R}$ el factor de resistencia igual a 0.90 y $V_{R}$ es la contribución mínima propuesta al cortante lateral resistente reportada en la tabla 3. Así, en la tabla 6, se presenta la sección transversal en el diseño de las columnas internas por flexión conforme al procedimiento descrito, donde $d$ es el peralte de la sección cajón, $t$ el espesor de la placa propuesto, $M_{p}$ el momento plástico resistente por la sección propuesta y $M_{\text {nec }}$ el momento actuante. 
Tabla 6. Prediseño de las columnas internas por flexión

\begin{tabular}{cccccccc}
\hline Nivel & $\begin{array}{c}D \\
(\mathrm{~cm})\end{array}$ & $\begin{array}{c}T \\
(\mathrm{~cm})\end{array}$ & $\begin{array}{c}\text { Tipo de } \\
\text { sección }\end{array}$ & $\begin{array}{c}Z \\
\left(\mathrm{~cm}^{3}\right)\end{array}$ & $\begin{array}{c}M_{p} \\
\text { (ton } \mathrm{m})\end{array}$ & $\begin{array}{c}\Sigma M_{p} \\
\text { (ton } \mathrm{m})\end{array}$ & $\begin{array}{c}M_{\text {nec }}=V_{R} h \\
\text { (ton m) }\end{array}$ \\
\hline 14 & 55 & 0.95 & 2 & 4163.43 & 131.82 & 4,746 & 373 \\
13 & 55 & 0.95 & 2 & 4163.43 & 131.82 & 4,746 & 874 \\
12 & 55 & 0.95 & 2 & 4163.43 & 131.82 & 4,746 & 1,335 \\
11 & 55 & 0.95 & 2 & 4163.43 & 131.82 & 4,746 & 1,759 \\
10 & 55 & 1.91 & 1 & 8078.62 & 255.79 & 9,208 & 2,143 \\
9 & 55 & 1.91 & 1 & 8078.62 & 255.79 & 9,208 & 2,490 \\
8 & 55 & 1.91 & 1 & 8078.62 & 255.79 & 9,208 & 2,798 \\
7 & 55 & 1.91 & 1 & 8078.62 & 255.79 & 9,208 & 3,067 \\
6 & 55 & 1.91 & 1 & 8078.62 & 255.79 & 9,208 & 3,298 \\
5 & 55 & 2.54 & 1 & 10493.51 & 332.25 & 11,961 & 3,490 \\
4 & 55 & 2.54 & 1 & 10493.51 & 332.25 & 11,961 & 3,644 \\
3 & 55 & 2.54 & 1 & 10493.51 & 332.25 & 11,961 & 3,760 \\
2 & 55 & 2.54 & 1 & 10493.51 & 332.25 & 11,961 & 3,837 \\
1 & 55 & 2.54 & 1 & 10493.51 & 332.25 & 11,961 & 3,875 \\
\hline
\end{tabular}

\section{Prediseño por carga axial de las columnas internas}

Las columnas adyacentes a las crujías contraventeadas fueron prediseñadas para soportar una carga axial $P_{k}$ determinada con la ecuación 2, que considera el incremento de carga axial por la respuesta inelástica de los contraviento.

En la tabla 7 se presenta la determinación de la carga actuante para la aplicación de la metodología expuesta, donde $P_{\text {grav }}$ es la carga axial provocado por las cargas gravitacionales, $P_{R}$ contr y $T_{R}$ Contr son la carga de plastificación de los contravientos $P_{f}$ por pandeo en compresión y por fluencia en tensión determinados conforme a las NTCM-04.

Entonces, la componente de la carga que axializará la columna se obtiene afectando la aportación de la carga de plastificación de los contravientos $P_{f}$ con un factor $f_{i}$ que se determina con la ecuación 3 . En la tabla 8, se presenta el factor de reducción determinado para el modelo diseñado.

Para ejemplificar el procedimiento, suponga que desea determinar la componente de contribución que soportará una columna noveno piso. Entonces, deberá sumar las cargas de plastificación de los contravientos por entrepiso afectadas por el factor $f_{i}$ reportado en la columna 6 de la tabla 8, de manera que la contribución de la carga de plastificación del contraviento del piso 9 se considera al $100 \%$, del piso 10 al 98\%, del piso 11 al 92\% y así sucesivamente, hasta que la aportación del piso 14 es al 60\%.

Finalmente, la carga axial resistente de las columnas $R_{c o l}$ de inestabilidad por flexión se determina con la ecuación 11, de donde se obtiene el área transversal necesaria.

$R_{\text {Col }}=\frac{F_{R} A_{t} F_{y}}{\left(1+\lambda^{2 n}-0.15^{2 n}\right)^{1 / n}}$

En la tabla 9 se presenta la revisión de la sección propuesta considerando la carga axial actuante en las columnas internas; esta carga resistente debe ser mayor a la carga actuante $P_{k}$ de la tabla 7 . En la determinación de la resistencia a compresión se consideró $n=1.4$ siguiendo el planteamiento de las NTCM-04 con una longitud igual a $L=350 \mathrm{~cm}$. 
Tabla 7. Determinación de la carga axial actuante del prediseño de las columnas internas por carga axial

\begin{tabular}{ccccccc}
\hline \multicolumn{7}{c}{ Cargas de fluencia de los } \\
Nivel & \multicolumn{2}{c}{ contravientos } & $\Sigma P_{f} f_{i}$ & $\operatorname{sen} \theta\left(\Sigma P_{f .} f_{i}\right)$ & $P_{\text {grav }}$ & $P_{k}$ \\
& $P_{R \text { Contr }}$ & $T_{R \text { Contr }}$ & & & & \\
& $(\mathrm{kg})$ & $(\mathrm{kg})$ & $($ ton $)$ & $($ ton $)$ & (ton) & (ton) \\
\hline 14 & 57,718 & 76,595 & 76.59 & 54.2 & 38.71 & 102.16 \\
13 & 57,718 & 76,595 & 147.11 & 104.0 & 94.57 & 175.87 \\
12 & 75,805 & 101,194 & 238.95 & 200.2 & 150.43 & 281.72 \\
11 & 75,805 & 101,194 & 325.49 & 258.8 & 206.29 & 346.15 \\
10 & 75,805 & 101,194 & 405.80 & 312.9 & 262.15 & 405.60 \\
9 & 75,805 & 101,194 & 479.33 & 362.1 & 318.01 & 521.16 \\
8 & 92,244 & 123,831 & 568.15 & 496.9 & 373.87 & 730.91 \\
7 & 92,244 & 123,831 & 648.53 & 543.8 & 429.73 & 843.93 \\
6 & 92,244 & 123,831 & 719.02 & 583.1 & 485.59 & 948.60 \\
5 & 92,244 & 123,831 & 778.20 & 614.0 & 541.45 & 1044.02 \\
4 & 109,228 & 147,527 & 848.10 & 757.0 & 38.71 & 1262.80 \\
3 & 109,228 & 147,527 & 902.35 & 769.6 & 446.88 & 1338.11 \\
2 & 109,228 & 147,527 & 936.88 & 766.9 & 502.74 & 1396.56 \\
1 & 109,228 & 147,527 & 948.17 & 748.2 & 558.60 & 1437.51 \\
\hline
\end{tabular}

Tabla 8. Factor de reducción de la carga de plastificación de los contravientos $f_{i}$

\begin{tabular}{|c|c|c|c|c|c|c|c|c|c|c|c|c|c|c|}
\hline \multirow{2}{*}{$\begin{array}{c}S_{n} \text { entrepiso donde } \\
\text { se determina la } \\
\text { carga axial }\end{array}$} & \multicolumn{14}{|c|}{$S_{i}$ entrepiso donde se está determinando el factor de reducción } \\
\hline & 14 & 13 & 12 & 11 & 10 & 9 & 8 & 7 & 6 & 5 & 4 & 3 & 2 & 1 \\
\hline 14 & 0.00 & 0.05 & 0.13 & 0.21 & 0.29 & 0.37 & 0.44 & 0.52 & 0.60 & 0.68 & 0.76 & 0.84 & 0.92 & 1.00 \\
\hline 13 & 0.05 & 0.14 & 0.22 & 0.31 & 0.39 & 0.48 & 0.56 & 0.64 & 0.73 & 0.81 & 0.89 & 0.96 & 1.00 & \\
\hline 12 & 0.14 & 0.23 & 0.32 & 0.41 & 0.50 & 0.58 & 0.67 & 0.75 & 0.83 & 0.91 & 0.97 & 1.00 & & \\
\hline 11 & 0.22 & 0.32 & 0.41 & 0.51 & 0.60 & 0.68 & 0.77 & 0.85 & 0.92 & 0.97 & 1.00 & & & \\
\hline 10 & 0.31 & 0.41 & 0.51 & 0.60 & 0.69 & 0.78 & 0.86 & 0.93 & 0.98 & 1.00 & & & & \\
\hline 9 & 0.39 & 0.50 & 0.60 & 0.69 & 0.78 & 0.86 & 0.93 & 0.98 & 1.00 & & & & & \\
\hline 8 & 0.48 & 0.58 & 0.68 & 0.78 & 0.86 & 0.93 & 0.98 & 1.00 & & & & & & \\
\hline 7 & 0.56 & 0.67 & 0.77 & 0.86 & 0.93 & 0.98 & 1.00 & & & & & & & \\
\hline 6 & 0.64 & 0.75 & 0.85 & 0.93 & 0.98 & 1.00 & & & & & & & & \\
\hline 5 & 0.73 & 0.83 & 0.92 & 0.98 & 1.00 & & & & & & & & & \\
\hline 4 & 0.81 & 0.91 & 0.97 & 1.00 & & & & & & & & & & \\
\hline 3 & 0.89 & 0.97 & 1.00 & & & & & & & & & & & \\
\hline 2 & 0.96 & 1.00 & & & & & & & & & & & & \\
\hline 1 & 1.00 & & & & & & & & & & & & & \\
\hline
\end{tabular}

\section{Prediseño de las columnas externas}

Dado que las columnas perimetrales tienen una carga axial menor, siguiendo las tendencias de diseño de la práctica, se propuso una reducción en el espesor de las placas manteniendo el peralte de la sección en las columnas externas de la edificación. En la tabla 10 se reportan las secciones finales consideradas después de realizar el procedimiento para el prediseño por flexión y carga axial descrito en el apartado anterior. 
Tabla 9 Determinación de la carga axial del prediseño de las columnas internas por carga axial

\begin{tabular}{cccccccc}
\hline Nivel & $\begin{array}{c}A_{t \text { Col }} \\
\mathrm{cm}^{2}\end{array}$ & $\begin{array}{c}P_{y} \\
\text { Ton }\end{array}$ & $\begin{array}{c}I \\
\mathrm{~cm}^{4}\end{array}$ & $\begin{array}{c}r \\
\mathrm{~cm}\end{array}$ & $K L / r$ & $\lambda$ & $\begin{array}{c}P_{\text {resist }} \\
\text { Ton }\end{array}$ \\
\hline 14 & 205.4 & 722.6 & 100,035 & 22.07 & 15.9 & 0.21 & 646.8 \\
13 & 205.4 & 722.6 & 100,035 & 22.07 & 15.9 & 0.21 & 646.8 \\
12 & 205.4 & 722.6 & 100,035 & 22.07 & 15.9 & 0.21 & 646.8 \\
11 & 205.4 & 722.6 & 100,035 & 22.07 & 15.9 & 0.21 & 646.8 \\
10 & 405.6 & $1,426.9$ & 190,784 & 21.69 & 16.1 & 0.21 & $1,276.7$ \\
9 & 405.6 & $1,426.9$ & 190,784 & 21.69 & 16.1 & 0.21 & $1,276.7$ \\
8 & 405.6 & $1,426.9$ & 190,784 & 21.69 & 16.1 & 0.21 & $1,276.7$ \\
7 & 405.6 & $1,426.9$ & 190,784 & 21.69 & 16.1 & 0.21 & $1,276.7$ \\
6 & 405.6 & $1,426.9$ & 190,784 & 21.69 & 16.1 & 0.21 & $1,276.7$ \\
5 & 532.9 & $1,875.1$ & 245,044 & 21.44 & 16.3 & 0.22 & $1,677.1$ \\
4 & 532.9 & $1,875.1$ & 245,044 & 21.44 & 16.3 & 0.22 & $1,677.1$ \\
3 & 532.9 & $1,875.1$ & 245,044 & 21.44 & 16.3 & 0.22 & $1,677.1$ \\
2 & 532.9 & $1,875.1$ & 245,044 & 21.44 & 16.3 & 0.22 & $1,677.1$ \\
1 & 532.9 & $1,875.1$ & 245,044 & 21.44 & 16.3 & 0.22 & $1,677.1$ \\
\hline
\end{tabular}

\section{Prediseño por balance de resistencias en el nodo}

Además, se revisó que las capacidades a flexión entre las trabes y las columnas prediseñadas tuvieran una proporción de al menos $\alpha=1.20$, conforme a la inecuación 4 (inciso $g$ de la figura 1). En la tabla 11 se presenta la suma de los momentos plásticos de las columnas (entrepiso superior y entrepiso inferior) y la suma de los momentos de las trabes que convergen a un mismo nudo crítico, a partir de las secciones transversales del prediseño. La tabla distingue las capacidades de los marcos perimetrales y los marcos internos, porque las columnas tienen diferente sección transversal.

Tabla 10. Prediseño de las columnas perimetrales

\begin{tabular}{cccccccc}
\hline Nivel & $\begin{array}{c}d \\
\mathrm{~cm}\end{array}$ & $\begin{array}{c}t \\
\mathrm{~cm}\end{array}$ & $\begin{array}{c}\text { Tipo de } \\
\text { sección }\end{array}$ & $\begin{array}{c}Z \\
\mathrm{~cm}^{3}\end{array}$ & $\begin{array}{c}M_{p} \\
\text { ton } \mathrm{m}\end{array}$ & $\begin{array}{c}\Sigma M_{\pi} \\
\text { ton } \mathrm{m}\end{array}$ & $\begin{array}{c}M_{\text {nec }}=V_{R} h \\
\text { ton } \mathrm{m}\end{array}$ \\
\hline 14 & 55 & 0.95 & 2 & $4,163.4$ & 131.8 & 4,746 & 373 \\
13 & 55 & 0.95 & 2 & $4,163.4$ & 131.8 & 4,746 & 874 \\
12 & 55 & 0.95 & 2 & $4,163.4$ & 131.8 & 4,746 & 1,335 \\
11 & 55 & 0.95 & 2 & $4,163.4$ & 131.8 & 4,746 & 1,759 \\
10 & 55 & 1.27 & 1 & $5,500.6$ & 174.2 & 6,270 & 2,143 \\
9 & 55 & 1.27 & 1 & $5,500.6$ & 174.2 & 6,270 & 2,490 \\
8 & 55 & 1.27 & 1 & $5,500.6$ & 174.2 & 6,270 & 2,798 \\
7 & 55 & 1.27 & 1 & $5,500.6$ & 174.2 & 6,270 & 3,067 \\
6 & 55 & 1.27 & 2 & $5,500.6$ & 174.2 & 6,270 & 3,298 \\
5 & 55 & 2.22 & 1 & $9,281.9$ & 293.8 & 10,580 & 3,490 \\
4 & 55 & 2.22 & 1 & $9,281.9$ & 293.8 & 10,580 & 3,644 \\
3 & 55 & 2.22 & 1 & $9,281.9$ & 293.8 & 10,580 & 3,760 \\
2 & 55 & 2.22 & 1 & $9,281.9$ & 293.8 & 10,580 & 3,837 \\
1 & 55 & 2.22 & 1 & $9,281.9$ & 293.8 & 10,580 & 3,875 \\
\hline
\end{tabular}




\section{ANÁLISIS SÍSMICO}

La estructura se supuso ubicada en la zona IIIa de la subzonificación de las Normas Técnicas para el Diseño por Sismo (Ap. 3, NTCS-04), asociada a un periodo fundamental del suelo $T_{s}=1.05$ seg. Entonces, siguiendo lo establecido en el Apéndice A (NTCS), los periodos característicos resultaron $T_{a}=$ 0.558 seg y $T_{b}=1.35 \mathrm{seg}$; un coeficiente símico $c=0.786$; un coeficiente de aceleración del terreno $a_{0}=$ 0.1825 y un cociente $k=0.95$.

Tabla 11. Comprobación de capacidades por flexión de trabes y columnas

\begin{tabular}{|c|c|c|c|c|c|c|c|c|}
\hline \multirow{3}{*}{ Nivel } & \multirow{2}{*}{\multicolumn{3}{|c|}{$\begin{array}{l}\text { Módulo de sección plástico, } \\
\qquad \mathrm{Z}\left(\mathrm{cm}^{3}\right)\end{array}$}} & \multicolumn{3}{|c|}{ Suma de momentos plásticos, (ton m) } & \multirow{2}{*}{\multicolumn{2}{|c|}{$\begin{array}{c}\text { Parámetro } \alpha \\
\text { (debe cumplir } \alpha \geq 1.2 \text { ) }\end{array}$}} \\
\hline & & & & $\Sigma Z_{c} F_{y c}$ & $\Sigma Z_{c} F_{y c}$ & $Z_{v} F_{y v}$ & & \\
\hline & $\begin{array}{c}\text { Columna } \\
\text { perimetral }\end{array}$ & $\begin{array}{c}\text { Columna } \\
\text { interna }\end{array}$ & Trabe & $\begin{array}{c}\text { Columna } \\
\text { perimetral }\end{array}$ & $\begin{array}{c}\text { Columna } \\
\text { interna }\end{array}$ & $\begin{array}{c}\text { Trabe } \\
\text { principal }\end{array}$ & $\begin{array}{c}\text { Nodo } \\
\text { perimetral }\end{array}$ & $\begin{array}{l}\text { Nodo } \\
\text { externo }\end{array}$ \\
\hline 14 & 4163.4 & 4163.4 & 1655.0 & 292.9 & 292.9 & 83.7 & 3.50 & 3.50 \\
\hline 13 & 4163.4 & 4163.4 & 1835.0 & 292.9 & 292.9 & 92.9 & 3.15 & 3.15 \\
\hline 12 & 4163.4 & 4163.4 & 1835.0 & 292.9 & 292.9 & 92.9 & 3.15 & 3.15 \\
\hline 11 & 4163.4 & 4163.4 & 1835.0 & 292.9 & 292.9 & 92.9 & 3.15 & 3.15 \\
\hline 10 & 8078.6 & 5500.6 & 1835.0 & 568.4 & 387.0 & 92.9 & 6.12 & 4.17 \\
\hline 9 & 8078.6 & 5500.6 & 1835.0 & 568.4 & 387.0 & 92.9 & 6.12 & 4.17 \\
\hline 8 & 8078.6 & 5500.6 & 1835.0 & 568.4 & 387.0 & 92.9 & 6.12 & 4.17 \\
\hline 7 & 8078.6 & 5500.6 & 2016.0 & 568.4 & 387.0 & 102.0 & 5.57 & 3.79 \\
\hline 6 & 8078.6 & 5500.6 & 2016.0 & 568.4 & 387.0 & 102.0 & 5.57 & 3.79 \\
\hline 5 & 10493.5 & 9281.9 & 2016.0 & 738.3 & 653.1 & 102.0 & 7.24 & 6.40 \\
\hline 4 & 10493.5 & 9281.9 & 2016.0 & 738.3 & 653.1 & 102.0 & 7.24 & 6.40 \\
\hline 3 & 10493.5 & 9281.9 & 2016.0 & 738.3 & 653.1 & 102.0 & 7.24 & 6.40 \\
\hline 2 & 10493.5 & 9281.9 & 2016.0 & 738.3 & 653.1 & 102.0 & 7.24 & 6.40 \\
\hline 1 & 10493.5 & 9281.9 & 2016.0 & 738.3 & 653.1 & 102.0 & 7.24 & 6.40 \\
\hline
\end{tabular}

Considerando la relación de esbeltez del modelo es igual a $H / B=1.40$ y que el periodo aproximado de la estructura es $T=0.08 n=1.12 \mathrm{seg}$, el factor por comportamiento no lineal es igual a $Q=2.33$ (ecuación 7), mientras que el factor por sobrerresistencia es igual a $R=4.5$ (ecuación 8) siguiendo los incisos $h$ e $i$ de la figura 1 .

\section{Condiciones de regularidad}

No fue necesario hacer alguna modificación por irregularidad de la estructura (inciso $j$ de la figura 1), debido a que el modelo satisface todos los requisitos para considerarse como regular (Ap. 6.1, NTCS04).

\section{Espectros de diseño}

Así, siguiendo el criterio del Apéndice A, el factor de comportamiento sísmico reducido con fines de diseño es igual a $Q^{\prime}=2.368$ (ecuación 12), de manera que en la figura 8 se ilustra el espectro de diseño elástico obtenido conforme al planteamiento del Apéndice A (NTCS-04) y el espectro de diseño inelástico considerando los factores de reducción por comportamiento no lineal y por sobrerresistencia determinados anteriormente (inciso $k$ de la figura 1 ). 


$$
\begin{array}{lc}
Q^{\prime}=1+(Q-1) \sqrt{\frac{\beta}{k} \frac{T}{T_{a}}} & \text { si } T<T_{a} \\
Q^{\prime}=1+(Q-1) \sqrt{\frac{\beta}{k}} & \text { si } T_{a} \leq T<T_{b} \\
Q^{\prime}=1+(Q-1) \sqrt{\frac{\beta p}{k}} & \text { si } T_{b} \leq T
\end{array}
$$

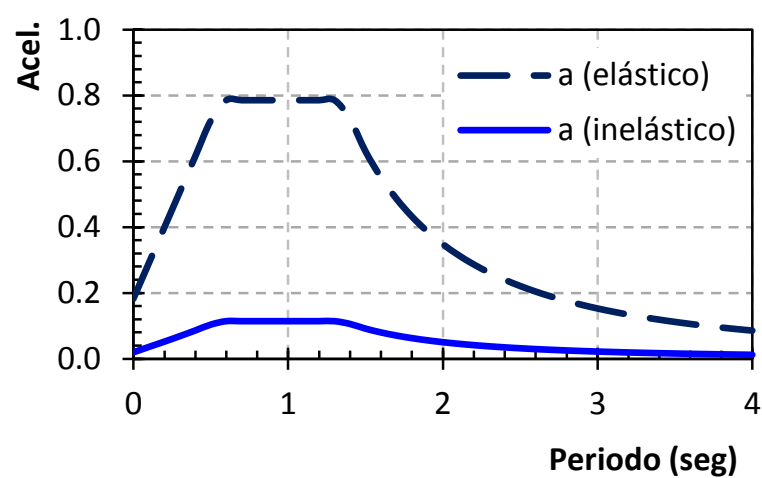

Figura 8. Espectros de diseño elástico e inelástico para $T_{s}=1.05$ seg y $Q=2.33$ según el Apéndice A

\section{ANÁLISIS MODAL ESPECTRAL}

Con las secciones transversales propuestas en la etapa de prediseño, se realizó un análisis modal espectral en tres dimensiones considerando los lineamientos del Apéndice A (NTCS-04). Las relaciones de esfuerzo (proporción entre el esfuerzo actuante y el esfuerzo resistente de las secciones estructurales) obtenidas concuerdan con la filosofía de diseño supuesta desde la etapa de diseño (columna fuerte - viga débil - contraviento más débil), como se ilustra en la figura 9. En las gráficas, se aprecia ligeramente el cambio de sección transversal de los contravientos y columnas sin que éstos se asocien a cambios significativos en la resistencia.

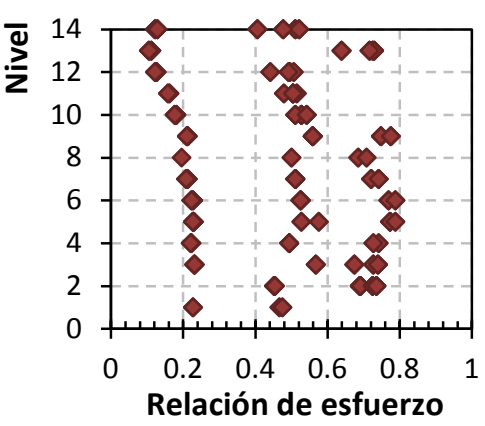

Contravientos

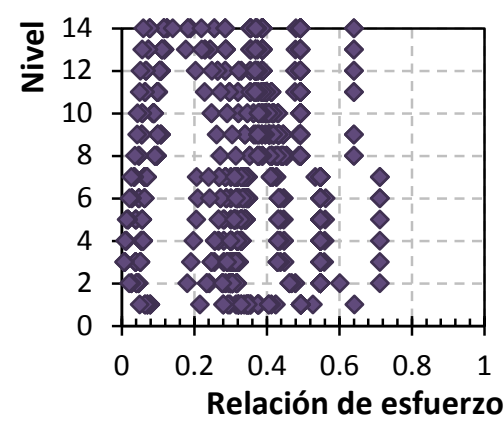

Trabes

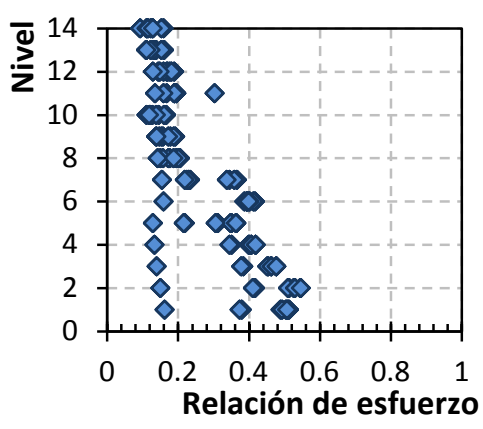

Columnas

Figura 9. Relaciones de esfuerzos del modelo de 14 niveles

Los periodos obtenidos en el análisis se presentan en la tabla 12, mientras que en la figura 10 se ha incluido la configuración de los primeros tres modos. El periodo fundamental es igual a $T=1.209$ seg, mayor al que se había supuesto inicialmente $(T=0.08 n=1.12 \mathrm{seg})$, siguiendo el inciso $l$ de la figura 1 . Sin 
embargo, este aumento no afecta significativamente el espectro de diseño, porque este periodo fundamental está en el mismo intervalo que define el factor de reducción por sobrerresistencia, es decir, que se mantiene $R=4.5$ y también $Q=2.33$, debido a que no es función del periodo fundamental $\left(Q^{\prime}=\right.$ 2.368).

Tabla 12. Periodos y aportaciones modales del modelo de 14 niveles

\begin{tabular}{ccccc}
\hline Modo & $\begin{array}{c}\text { Periodo } \\
\text { (seg) }\end{array}$ & RX & RY & RZ \\
\hline 1 & 1.209 & 0.00 & 97.40 & 0.00 \\
2 & 1.208 & 97.41 & 0.00 & 0.00 \\
3 & 0.938 & 0.00 & 0.00 & 71.30 \\
\hline
\end{tabular}

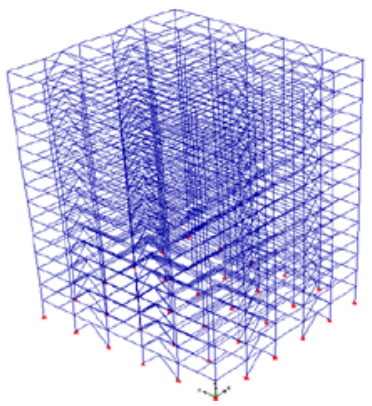

Primer modo

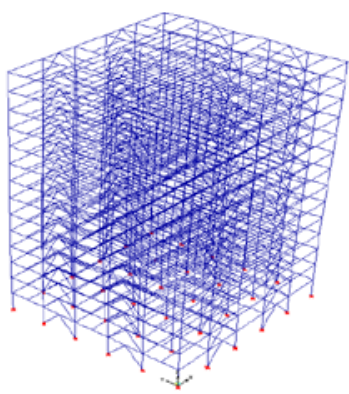

Segundo modo

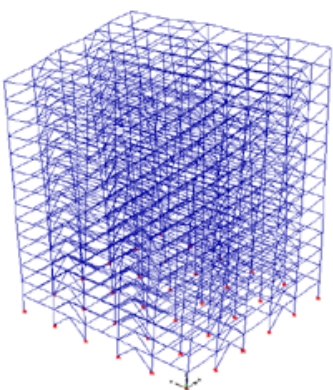

Tercer modo

Figura 10. Configuración de los primeros tres modos del modelo de 14 niveles

\section{Balance entre rigidez lateral y resistencia}

En la figura 11 se reporta el comportamiento de la rigidez lateral y la resistencia lateral en cada uno de los entrepisos que conforman la estructura considerando los elementos estructurales modelados. La resistencia lateral se determinó con los resultados del análisis, mientras que la rigidez se calculó distinguiendo la aportación de las columnas y el sistema de contravientos.

Así, mediante el uso de los elementos estructurales propuestos siguiendo la metodología propuesta, se satisface ampliamente la resistencia lateral mínima del 61.4\% para un modelo de 14 niveles, que deben contribuir las columnas del marco en relación a la aportación del sistema de contravientos (inciso $m$ de la figura 1).

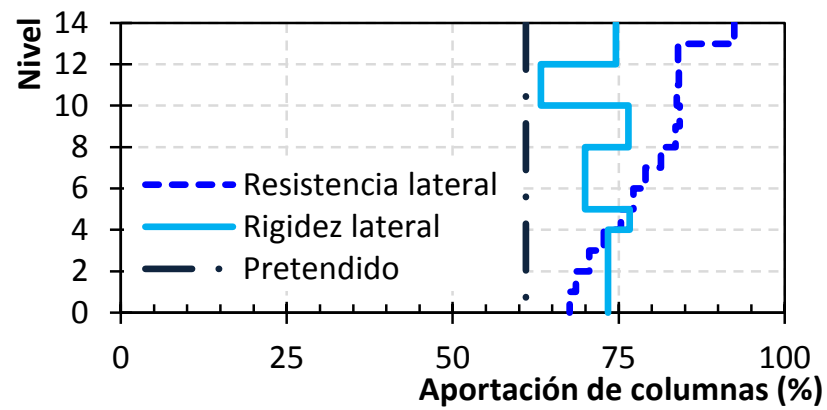

Figura 11. Comportamiento de la rigidez lateral y cortante lateral resistente del modelo 
Finalmente, en la tabla 13 se reportan las secciones finales para el modelo de 14 niveles. Las columnas son de sección cajón con una sección para las columnas de los marcos de la periferia de la estructura y las columnas de los marcos interiores. Los contravientos se modelaron igualmente de sección cajón, mientras que las trabes son de sección comercial IR.

Tabla 13. Secciones transversales del modelo

\begin{tabular}{|c|c|c|c|c|}
\hline \multirow{2}{*}{ Nivel } & \multicolumn{2}{|c|}{ Columnas } & \multirow{2}{*}{ Trabes } & \multirow{2}{*}{ Contravientos } \\
\hline & Perimetrales & Internas & & \\
\hline 14 & OC 55x0.95 cm & OC 55x0.95 cm & W $18 x 82.0 \mathrm{~kg} / \mathrm{m}$ & OC $18 x 0.48 \mathrm{~cm}$ \\
\hline 13 & OC $55 \times 0.95 \mathrm{~cm}$ & OC $55 \times 0.95 \mathrm{~cm}$ & W 18x96.7 kg/m & OC $18 \times 0.48 \mathrm{~cm}$ \\
\hline 12 & OC $55 \times 0.95 \mathrm{~cm}$ & OC $55 \times 0.95 \mathrm{~cm}$ & W 18x96.7 kg/m & OC $18 \times 0.64 \mathrm{~cm}$ \\
\hline 11 & OC $55 \times 0.95 \mathrm{~cm}$ & OC $55 \times 0.95 \mathrm{~cm}$ & W 18x96.7 kg/m & OC $18 \times 0.64 \mathrm{~cm}$ \\
\hline 10 & OC $55 \times 1.27 \mathrm{~cm}$ & OC $55 \times 1.91 \mathrm{~cm}$ & W 18x96.7 kg/m & OC $18 \times 0.64 \mathrm{~cm}$ \\
\hline 9 & OC $55 \times 1.27 \mathrm{~cm}$ & OC $55 \times 1.91 \mathrm{~cm}$ & W 18x96.7 kg/m & OC $18 \times 0.64 \mathrm{~cm}$ \\
\hline 8 & OC 55x1.27 cm & OC 55x1.91 cm & W 18x96.7 kg/m & OC $18 \times 0.79 \mathrm{~cm}$ \\
\hline 7 & OC 55x1.27 cm & OC $55 \times 1.91 \mathrm{~cm}$ & W 18x105.3 kg/m & OC $18 \times 0.79 \mathrm{~cm}$ \\
\hline 6 & OC $55 \times 1.27 \mathrm{~cm}$ & OC $55 \times 1.91 \mathrm{~cm}$ & W $18 x 105.3$ kg/m & OC $18 \times 0.79 \mathrm{~cm}$ \\
\hline 5 & OC $55 \times 2.22 \mathrm{~cm}$ & OC $55 \times 2.54 \mathrm{~cm}$ & W $18 \times 105.3 \mathrm{~kg} / \mathrm{m}$ & OC $18 \times 0.79 \mathrm{~cm}$ \\
\hline 4 & OC 55x2.22 cm & OC $55 \times 2.54 \mathrm{~cm}$ & W $18 x 105.3 \mathrm{~kg} / \mathrm{m}$ & OC $18 \times 0.95 \mathrm{~cm}$ \\
\hline 3 & OC 55x2.22 cm & OC $55 \times 2.54 \mathrm{~cm}$ & W 18x105.3 kg/m & OC $18 \times 0.95 \mathrm{~cm}$ \\
\hline 2 & OC 55x2.22 cm & OC $55 \times 2.54 \mathrm{~cm}$ & W 18x105.3 kg/m & OC $18 \times 0.95 \mathrm{~cm}$ \\
\hline 1 & OC 55x2.22 cm & OC55x2.54 cm & W 18x105.3 kg/m & OC $18 \times 0.95 \mathrm{~cm}$ \\
\hline
\end{tabular}

\section{Estados límite de deformación}

Las condiciones de simetría en rigidez impuestas originaron que la respuesta en ambas direcciones globales, la deformación sea muy similar como se reporta en la tabla 14, donde se presentan las distorsiones inelásticas máximas por entrepiso obtenidas en el análisis (inciso $n$ de la figura 1).

Tabla 14. Distorsiones inelásticas máximas (\%) obtenidas en el análisis del modelo

\begin{tabular}{ccc}
\hline Nivel & Distorsión en X & Distorsión en Y \\
\hline N14 & 0.27 & 0.27 \\
N13 & 0.34 & 0.34 \\
N12 & 0.37 & 0.37 \\
N11 & 0.41 & 0.41 \\
N10 & 0.42 & 0.42 \\
N09 & 0.44 & 0.44 \\
N08 & 0.42 & 0.42 \\
N07 & 0.41 & 0.41 \\
N06 & 0.40 & 0.40 \\
N05 & 0.38 & 0.38 \\
N04 & 0.34 & 0.34 \\
N03 & 0.31 & 0.31 \\
N02 & 0.27 & 0.27 \\
N01 & 0.15 & 0.15 \\
\hline
\end{tabular}


Conforme al Apéndice A (NTCS-04), la revisión de limitación de daños solicita que las distorsiones obtenidas del análisis estructural multiplicadas por el factor $Q^{\prime} R / 7=1.5223\left(Q^{\prime}=2.368\right.$ y $\left.R=4.5\right)$ sean menores que $\delta_{\text {perm }}=0.004$, como se presenta en la figura 12 .

Por otra parte, en la figura 13 se muestra que las distorsiones inelásticas multiplicadas por el factor $Q R=10.49$ obtenidas del análisis son menores al límite $\delta_{\text {perm }}=0.015$ (Tabla A.1, NTCS-04) para satisfacer el requisito para seguridad contra colapso en ambas direcciones globales.

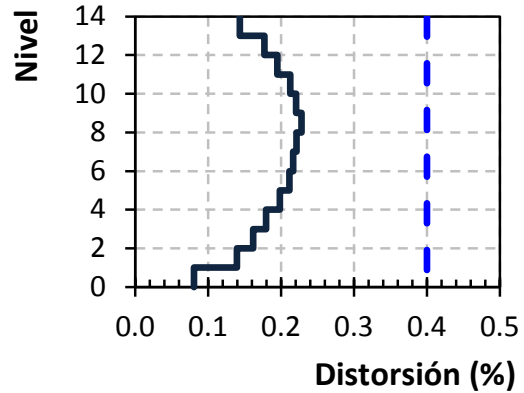

Dirección global X

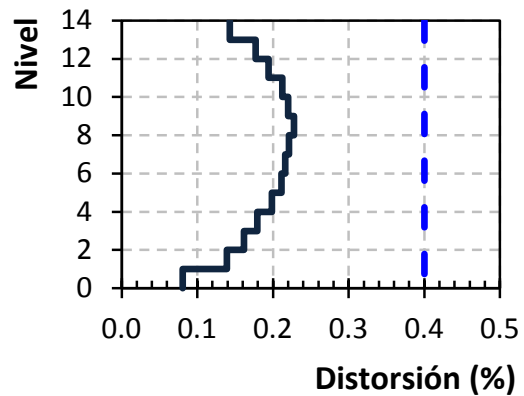

Dirección global Y

Figura 12. Revisión de distorsiones para limitación de daños a elementos no estructurales

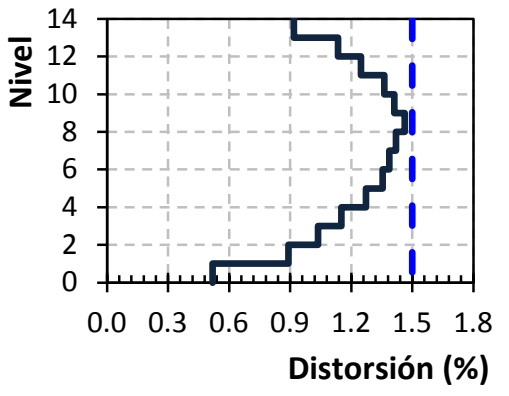

Dirección global X

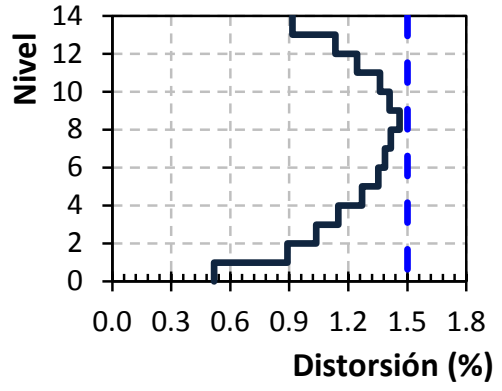

Dirección global Y

Figura 13. Revisión de distorsiones para seguridad contra colapso

\section{CONCLUSIONES}

En este artículo se presenta una metodología de diseño por capacidad para edificios regulares estructurados con marcos dúctiles de acero con contraventeo concéntrico, que pretende establecer los requisitos mínimos que deben ser considerados en el diseño de este sistema estructural, adaptados a los criterios de diseño sísmico de las NTC, para que los mecanismos de colapso desarrollados concuerden con las premisas iniciales de diseño de columna fuerte - viga débil - contraviento más débil con una adecuada distribución de la respuesta inelástica en la altura y redistribuciones de cargas predecibles.

Asimismo, el procedimiento de diseño, que se desarrolló a partir de los resultados obtenidos en Tapia y Tena (2011), tendencias de reglamentos internacionales, resultados de pruebas experimentales y otras investigaciones recientes, propone ecuaciones para la determinación de factores de reducción por ductilidad y sobrerresistencia más realistas para este sistema estructural y los requisitos que deben cumplir las secciones transversales de las columnas, trabes, contravientos y balances de rigidez en las conexiones. 
La metodología se ejemplificó, incluyendo un procedimiento sugerido de prediseño de las secciones transversales de los elementos, en un modelo de 14 niveles, que forma parte de un conjunto de edificios estructurados con marcos dúctiles de acero contraventeados situados en suelo blando con alturas entre los 10 a los 18 pisos (35 m a $63 \mathrm{~m}$ ), que se usaron para evaluar la propuesta. Los resultados obtenidos en el estudio para valorar la eficiencia del comportamiento inelástico de los edificios mencionados se presentan en artículo complementario Tapia y Tena (2013), los cuales demostraron que siguiendo el procedimiento de diseño por capacidad propuesto, los modelos tienen un comportamiento que concuerda razonablemente con las premisas iniciales de diseño discutidas en este artículo.

\section{REFERENCIAS}

Astaneh-Asl A, M Cochran y R Sabelli (2006), "Seismic detailing of gusset plates for special concentrically braced frames", Structural steel educational council, Technical information and product service. diciembre, www.steeltips.org.

Cochran, M y W Honeck (2004), "Design of special concentric braced frames (with comments on ordinary concentric braced frames)", Structural steel educational council, Technical information and product service, mayo, www.steeltips.org.

EC8-05 (2005), "EuroCode - 8. Design of structures for earthquake resistance. Part 1: General rules, seismic actions and rules for buildings”, European committee for standardization, Bruselas, marzo.

Godínez, E (2010), "Estudio del comportamiento de marcos dúctiles de concreto reforzado con contraventeo chevrón”, Tesis de Doctorado, Posgrado en Ingeniería Estructural, División de Ciencias Básicas e Ingeniería, Universidad Autónoma Metropolitana Azcapotzalco, julio.

Guilini-Charette, K (2009), “Effets des mouvements sismiques sur les structures en acier de la catégorie des constructions conventionnelles”. Tesis de maestría, Département des génies civil, géologique et des mines, École Polytechnique de Montréal, Canadá, diciembre.

Haddad, M y R Tremblay (2006), "Influence of connection design on the inelastic seismic response of HSS steel bracing members", Memorias, $11^{\text {th }}$ International Symposium and II International Conference on Tubular Structures, Quebec City, Canadá, septiembre. www.ists11.org.

Izvernari, C (2007), “The seismic behavior of steel braces with large sections”, Tesis de maestría, Département des Génies Civil, Géologique et des Mines, École Polytechnique de Montréal, Canadá, abril.

Izvernari, C, M Lacerte y R Tremblay (2007), “Seismic performance of multi-storey concentrically braced steel frames designed according to the 2005 Canadian seismic provisions”, Memorias, Ninth Canadian Conference on Earthquake Engineering, Ottawa, Ontario, Canadá, junio.

Lacerte, M y R Tremblay (2006), "Making use of brace overstrength to improve the seismic response of multistory split-X concentrically braced steel frames", Canadian Journal of Civil Engineering, Vol. 33, pp. 1005-1021.

Lundeen, T (2003), "Design and detailing of seismic connections for braced frame structures", Engineering Journal, American Institute of Steel Construction, Vol. 25, pp. 1-20.

Marino, E y M Nakashima (2005), "Seismic performance and new design procedure for chevron-braced frames”, Earthquake Engineering and Structural Dynamics, Vol. 34, pp. 434-452. doi: 10.1002/eqe.539. 
NTCCr-04, (2004), "Normas técnicas complementarias sobre criterios y acciones para el diseño estructural de las edificaciones”, Gaceta Oficial del Distrito Federal, décimo cuarta época, tomo II, octubre.

NTCM-04, (2004), "Normas técnicas complementarias sobre criterios y acciones para el diseño y construcción de estructuras metálicas”, Gaceta Oficial del Distrito Federal, décimo cuarta época, tomo II, octubre.

NTCS-04, (2004), “Normas técnicas complementarias para diseño por sismo”, Gaceta Oficial del Distrito Federal, décimo cuarta época, tomo II, octubre.

RCDF-2004, (2004), “Reglamento de construcciones para el Distrito Federal (RCDF)”. Gaceta Oficial del Distrito Federal, décima cuarta época, enero.

Tapia, E (2005), "Estudio de los criterios del RCDF-2004 y sus normas técnicas complementarias para el diseño sísmico de edificios regulares con base en marcos contraventeados de acero estructural”, Tesis de Maestría, División de Estudios de Postgrado de la Facultad de Ingeniería de la Universidad Nacional Autónoma de México, capítulo III, abril.

Tapia, E (2011), “Comportamiento de edificios regulares estructurados con marcos dúctiles de acero con contraventeo concéntrico en suelos blandos”, Tesis doctoral, Posgrado en Ingeniería Estructural, División de Ciencias Básicas e Ingeniería, Universidad Autónoma Metropolitana Azcapotzalco, julio.

Tapia-Hernández, E. y A. Tena-Colunga (2008), "Behavior of moment resisting concentrically braced frames (MRCBFs) of regular buildings in seismic zones”, Memorias, XIV World Conference of Earthquake Engineering, Beijing, China, Artículo No. 05-05-0008, CD-ROM, octubre.

Tapia, E y A Tena (2009), “Comportamiento sísmico de edificios regulares con marcos dúctiles de acero con contraventeo concéntrico diseñado conforme al reglamento del Distrito Federal mexicano", Revista Internacional de Ingeniería de Estructuras, Escuela Politécnica del Ejército. Vol. 13 y 14, No. 1, pp. 1-28, Quito, Ecuador.

Tapia, E y A Tena (2011), "Factores de ductilidad y sobrerresistencia en marcos de acero con contraventeo chevrón”, Revista de Ingeniería Sísmica, No. 84, pp. 47-68.

Tapia, E y A Tena (2013), “Diseño sísmico de marcos de acero contraventeados. Parte 2: Evaluación de la metodología”, Revista de Ingeniería Sísmica, No. 88, pp. 69-90.

Tapia, E, R Tremblay, A Tena, C Izvernari y M Lacerte (2009), "Estudio de la axialización de columnas en marcos de acero con contraventeo concéntrico en suelo firme”, Memorias, XVII Congreso Nacional de Ingeniería Sísmica, Puebla, Puebla, México, CDROM, noviembre.

Tremblay, R (2008), Comunicación personal.

Tremblay, R (2008a), "Influence of brace slenderness on the fracture life of rectangular tubular steel bracing members subjected to seismic inelastic loading”, Memorias, 2008 Structures Congress, American Society of Civil Engineers (ASCE), pp. 1-10, doi: 10.1061/41016(314)259. 